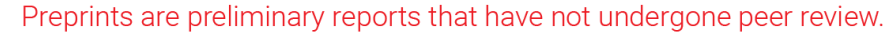 They should not be considered conclusive, used to inform clinical practice, or referenced by the media as validated information. \\ Cromolyn Platform Suppresses Fibrosis and Inflammation, Promotes Microglial Phagocytosis and Neurite Outgrowth
}

\section{Yi-Jun Wang}

AZTherapies, Inc

Matthew A. Downey

AZTherapies, Inc

\section{Sungwoon Choi}

Chungnam National University

Timothy M. Shoup

Massachusetts General Hospital, Harvard Medical School

David R. Elmaleh ( $\nabla$ delmaleh@mgh.harvard.edu )

Massachusetts General Hospital, Harvard Medical School

\section{Research Article}

Keywords: cromolyn, microglia, secretion, inflammatory, hmc3, proteins

Posted Date: August 26th, 2021

DOl: https://doi.org/10.21203/rs.3.rs-825684/v1

License: (c) (i) This work is licensed under a Creative Commons Attribution 4.0 International License.

Read Full License

Version of Record: A version of this preprint was published at Scientific Reports on November 12th, 2021.

See the published version at https://doi.org/10.1038/s41598-021-00465-6. 


\section{Abstract}

Neurodegenerative diseases are characterized by chronic neuroinflammation and may perpetuate ongoing fibrotic reactions within the central nervous system. In this report, RNA-seq analysis shows that administration of the pro-inflammatory cytokine TNF-a to HMC3 human microglia results in a robust upregulation of fibrosis-associated genes. Subsequent treatment with cromolyn and its fluorinated analogue F-cromolyn resulted in reduced secretion of collagen XVIII, fibronectin, and tenascin-c. Additionally, we show that cromolyn and F-cromolyn reduce secretion of pro-inflammatory proteins PLP1, PELP1, HSP90, IL-2, GRO-a, Eotaxin, and VEGF-A, while promoting secretion of anti-inflammatory IL-4 in HMC3 microglia. Neurite outgrowth in PC12 neuronal cells is augmented by cromolyn and F-cromolyn in concert with nerve growth factor. Treatment also differentially altered secretion of neurogenesis-related proteins TTL, PROX1, Rab35, and CSDE1 in HMC3 microglia. Finally, iPSC-derived human microglia more readily phagocytose $A \beta 42$ with cromolyn and F-cromolyn relative to controls. We propose the cromolyn platform targets multiple proteins upstream of PI3K/Akt/mTOR, NF-KB, and GSK-3 $\beta$ signaling pathways to affect cytokine, chemokine, and fibrosis-related protein expression.

\section{Introduction}

Most neurodegenerative diseases, including Alzheimer's disease (AD), amyotrophic lateral sclerosis (ALS), multiple sclerosis (MS), and Parkinson's disease (PD), are generally characterized by the chronic activation of the innate immune system, neuronal damage, and neuroinflammation ${ }^{1}$. Neuroinflammation is driven in no small part by reactive microglia, the resident macrophages of the central nervous system (CNS). When exposed to pathogens or inflammatory mediators, microglia adopt an activated phenotype and further propagate inflammation by releasing a wide range of cytokines and chemokines into the cellular environment ${ }^{1,2}$. Microglia polarization is inherently dynamic ${ }^{3,4}$, with functional outcomes mutually dependent on cross-communication between other cell types within the CNS and, by extension, their collective signals with those in the periphery ${ }^{4,5}$. Additionally, microglia play integral surveillance roles in the CNS, including tissue repair ${ }^{5}$, remodeling of ECM proteins ${ }^{6,7}$, and maintenance of synapses and neuronal health ${ }^{8,9}$.

Inflammation always precedes fibrosis. In the event of an acute injury, pathogen infiltration, or chronic inflammation in CNS tissue, fibrotic mechanisms are employed to close the wound to contain the site of injury to shield neurons from further damage and infection ${ }^{10}$, resulting in the formation of a glial scar. Glial scars are the primary form of fibrosis within the CNS and their formation is mediated by microglia, astrocytes, and other local and infiltrating immune cells ${ }^{10,11}$, with the inner core of the scar composed of PDGFRß-expressing fibroblast-like, non-neural cells believed to be sourced from differentiated pericytes from the basement membrane of the brain vasculature ${ }^{12,13}$. Pericytes alone and in mixed glia culture activate translocation of NF-KB p65 after exposure to LPS, IL-1 $\beta$, and TNF- ${ }^{14}$, a signaling pathway activated in fibroblasts associated with progression of pulmonary fibrosis ${ }^{15}$. As mentioned earlier, microglia may induce inflammatory activation of many cell types, including astrocytes. It was found that 
LPS-activated microglia secreted TNF, IL-1a, and C1q to induce A1 astrocyte activation, a neuroinflammatory state upregulated in the aging brain directly associated with the death of axotomized neurons ${ }^{16,17}$. In kind with their prominent surveillance roles in the CNS, microglia pro-inflammatory activation is well known to attract a variety of leukocytes to sites of inflammation, including $T$ cells ${ }^{9}$. It was found that the severity of fibrotic scarring in an autoimmune encephalomyelitis (EAE) mouse model caused by collagen-depositing fibroblasts was regulated by $T$ cell IFN- $\gamma$ signaling ${ }^{18}$. Microglia readily secrete IP-10 during inflammation ${ }^{19}$ and also express its receptor, CXCR3, that is necessary for microglial recruitment to sites of CNS injury ${ }^{20}$. T-cells also express CXCR3 and CCR5, the latter of which is a receptor for MIP-1 $a$ and MIP-1 $\beta$, and both act to increase T-cell recruitment to sites of inflammation ${ }^{21}$. It is possible that chronic activation of microglia to an inflammatory phenotype may lead to upregulation of innate CNS fibrotic mechanisms that are detrimental to brain parenchymal function. Therefore, modulating microglial behavior more toward an anti-inflammatory, anti-fibrotic, and phagocytic profile would be of meaningful benefit to limit neurodegeneration.

The aging brain has characteristic structural and physiological changes that been associated to varying degrees of age-related decline, including regional thickening of the basement membrane ${ }^{22}$ and differential changes in extracellular matrix (ECM) protein deposition ${ }^{23}$. Though the ECM provides critical structure to brain parenchyma to maintain stable, functional neuronal networks, studies with rat hippocampal neuron cultures suggest that reduction of ECM by hyaluronidase treatment encourages mature neurons to explore and redefine network connectivity, without inducing hyperexcitability even in the presence of bicuculline ${ }^{24}$. Early upregulation of ECM proteins in the hippocampus of APPswe transgenic $A D$ mice were discovered to coincide with increased $A \beta$ levels in the brain, hippocampal synapse impairment, and cognitive deficits; and hippocampal injection of an ECM inactivating chondroitinase ameliorated these deficits ${ }^{25}$. It is possible that dynamic structuring and restructuring of the ECM in the brain that accommodates neuronal activity is dysregulated in neurodegenerative disorders ${ }^{26}$, and the success of surveilling the ECM for aberrant structure may rely on maintaining antiinflammatory microglia ${ }^{27,28}$. Systemic inflammation modeled by daily peripheral injections of LPS in $\mathrm{MRL} / \mathrm{Ipr}$ mice shows that microglia migrate to the cerebral vasculature basement membrane in response to endothelial cell release of CCL $5^{29}$. This study revealed that microglia promote BBB integrity by expressing tight junction protein CLDN5 in early stages of inflammation but phagocytize astrocytic endfeet and promote BBB leakage in later stages of inflammation ${ }^{29}$. As astrocytes work in concert with microglia to contain and repair injuries in the $\mathrm{CNS}^{30}$, influx of peripheral cells due to decreased BBB integrity can trigger pattern recognition receptors (PRRs) in microglia, such as toll-like receptors (TLRs) and Nod-like receptors (NLRs), to worsen inflammation ${ }^{31}$. Some ECM components, including fibronectin, tenascin-c, and collagen, are upregulated during fibrosis of other tissues and are ligands to PRRs of microglia recognized as damage-associated molecular patterns (DAMPs) ${ }^{31-34}$. Macrophages differentiated from CD14 ${ }^{+}$blood monocytes have been shown to be a source of ECM components and ECM remodeling enzymes in the context of wound-healing in $\mathrm{skin}^{35}$. In our experiments we show that 
HMC3 microglia are capable of robust gene expression and protein secretion of key ECM proteins, including many collagens, classes of matrix metalloproteinases (MMPs), fibronectin, and tenascin-c.

Cromolyn is a mast cell stabilizer approved for the treatment of asthma as a dry powder, as well as in retinal solutions for eye and nasal applications. Cromolyn was recently evaluated in a Phase III clinical trial for early-onset Alzheimer's disease (AD) (NCT: NCT02547818-A Phase III Safety and Efficacy Study of ALZT-OP1 in Subjects with Evidence of Early Alzheimer's Disease) and is currently being evaluated in a Phase II clinical trial for amyotrophic lateral sclerosis (ALS) (NCT: (NCT04428775-A Phase II Safety and Biomarker Study of ALZT-OP1a in Subjects with Mild-Moderate ALS Disease). Cromolyn has been shown in vitro as an aggregation inhibitor of amyloid $\beta$-protein $(A \beta)$ as well as to reduce soluble levels of $A \beta$ in the mouse brain in vivo ${ }^{36}$. Additionally, cromolyn treatment to microglia promotes their migration to amyloid deposits and their subsequent anti-inflammatory phagocytosis ${ }^{37}$. Cromolyn has been shown to be well-tolerated in healthy volunteers and can achieve CSF levels that may be sufficient to inhibit aggregation of the daily amounts of brain amyloid produced ${ }^{38}$. Previous studies in our group have shown that cromolyn is able to reduce inflammatory cytokine and chemokine secretion by HMC3 microglia induced with TNF-a ${ }^{19}$. In the current study we utilized unbiased proteomic profiling of secreted proteins by HMC3 microglia exposed to TNF-a and RNA-sequencing to determine the anti-fibrotic potential of cromolyn and its fluorinated analog, F-cromolyn ${ }^{39}$, in neurodegenerative disease. Alongside cromolyn's ability to encourage anti-inflammatory profile of microglia, this study finds that cromolyn and F-cromolyn significantly affect TNF-a-induced HMC3 microglia expression of pro-fibrotic genes, key mediators of inflammation, amplify neurite outgrowth in PC12 neural cells in concert with nerve growth factor (NGF), and promote phagocytosis against A 342 of human iPSC-derived microglia. Altogether, the cromolyn platform represents a robust therapeutic strategy to address many aspects of neurodegenerative disease, particularly in multi-modal approaches that will be required to treat multi-faceted diseases like $A D$ and ALS.

\section{Methods And Materials}

\section{Chemicals and reagents}

DMEM-high glucose medium (Cat\#11995065), DMEM without phenol red (Cat\#21063029), RPMI 1640 Medium (Cat\#11875119), HS (horse serum) (Cat\#26050088), NGF (nerve growth factor) 2.5S Native Mouse Protein (Cat\#13257019), PBS-pH7.2 (Cat\#20012027), L-glutamine (Cat\#25030081), Trypsin-EDTA (Cat\#25200056), and penicillin-streptomycin (Cat\#15140122) were products of Gibco, Thermo Fisher Scientific. Goat anti-Rabbit IgG $(\mathrm{H}+\mathrm{L})$ Secondary Antibody-Alexa Flour 488 (Cat\#A11034), Hoechst33342 (Cat\#H3570), LysoTracker Red DND-99 (Cat\#L7528), and Pierce 16\% Formaldehyde (Cat\#28908) were products of Invitrogen, Thermo Fisher Scientific. FBS (fetal bovine serum) (Cat\#F4135), BSA (bovine serum albumin) (Cat\#A9647), DMSO (dimethyl sulfoxide) (Cat\#D2438), Triton X-100 (Cat\#T8787), and TWEEN-20 (Cat\#P1379) were purchased from Sigma-Aldrich. 
QIAsymphony RNA Kit (Cat\#931636) and Buffer RLT Plus (Cat\#1053393) were products of QIAGEN. Recombinant human TNF-a (Cat\#300-01A) was purchased from PeproTech. BD Matrigel Matrix (Cat\#354234) was purchased from BD Biosciences. B3-Tubulin (D71G9) Rabbit Antibody (Cat\#5568) was purchased from Cell Signaling Technology. Fluorescein (FITC)- $\beta$-Amyloid-42 (Cat\#A11191) was purchased from rPeptide. $3 \mathrm{~mL}$ Syringe/Needle Combination with Luer-Lok ${ }^{\mathrm{TM}}$ Tip (Cat\#8936G82), $13 \mathrm{~mm}$ syringe filter (PVDF, $0.22 \mu \mathrm{m}$ ) (Cat\#1159T77) were purchased from Thomas Scientific. Cromolyn and Fcromolyn were provided by AZTherapies and DMEM was used as diluent to achieve final concentrations as indicated.

\section{Cell line and cell culture}

HMC3 human microglial cell line (CRL-3304) and PC12 cell line (CRL-1721) were purchased from ATCC (American Type Culture Collection). HMC3 cells were cultured in DMEM medium with $10 \% \mathrm{FBS}, 1 \% \mathrm{~L}$ glutamine, and $1 \%$ penicillin/streptomycin (P/S), and were maintained in $37^{\circ} \mathrm{C}$ incubator at $5 \% \mathrm{CO}_{2} . \mathrm{PC} 12$ cells were cultured in RPMI-1640 medium supplemented with 10\% HS, 5\% FBS and 1\% P/S then placed in humidified air chamber containing $5 \% \mathrm{CO} 2$ at $37^{\circ} \mathrm{C}$. The monolayer cells were harvested by trypsin and seeded into microplates to assess compound effect on neurite outgrowth. Human iPSC-derived microglia (Cat\#BX-0900) was purchased from BrainXell.

\section{RNA-Sequencing analysis:}

\section{RNA isolation and sequencing}

Cells collected from each condition were lysed in the Buffer RLT Plus (QIAGEN), and RNA isolation were performed on a QIAsymphony SP/AS using QIAsymphony RNA Kit according to the manufacturer instruction. Quality check and quantification were then performed on the isolated RNA samples using Agilent Fragment Analyzer. RNA samples that passed quality check were used as input for sequencing library preparation, using Illumina stranded mRNA kit per the manufacturer instruction. Quality check and quantification were performed on the resulting libraries, followed by sequencing on an Illumina NextSeq 550 using NextSeq High Output 75 cycles kit and single read 75 bases format. Resulting raw data were converted and demultiplexed into fastq format using Illumina bc2fastq software. FastQC analyses were performed on the demultiplexed fastq files.

\section{Bioinformatics}

The fastq files resulting from sequencing were used as input for downstream analysis. Briefly, reads from fastq files were mapped to human genome (UCSC Hg38) with HISAT2 aligner, and read counting for genes were performed using featureCounts of Subread package. The results from featureCounts were used as input for differential gene expression and functional analysis in R. Differential expression analysis was performed with DESeq2. Genes that are differentially expressed are identified ( $p$-adj $<0.05$ with or without a 2-fold differences cutoff). The list of differentially expressed genes were used as input for functional analysis, including Gene Set Enrichment Analysis comparing against Molecular Signature Database (MSigDB, BROAD Institute) and over-representation analysis against Reactome database. Data 
analysis and visualization (dotplot, volcano plot, etc) were performed with $\mathrm{R}$, using DESeq2, fgsea, ReactomePA, clusterProliler and EnhancedVolcano packages.

\section{Proteomics Analysis using HRM-ID + Mass Spectrometry: \\ Sample preparation}

Proteins from $800 \mu \mathrm{l}$ of each sample were precipitated with acetone overnight at $-20^{\circ} \mathrm{C}$. Proteins were then denatured using Denature Buffer (Biognosys), and reduced and alkylated using Reduction and Alkylation Solution (Biognosys) for $60 \mathrm{~min}$ at $37^{\circ} \mathrm{C}$. Subsequently, digestion to peptides was carried out using trypsin (Promega, 1:50 protease to total protein ratio) overnight at $37^{\circ} \mathrm{C}$.

\section{Clean-up for mass spectrometry}

Peptides were desalted using BioPureSPN C18 MINI spin columns (The Nest Group) according to the manufacturer's instructions and dried down using a SpeedVac system. Peptides were resuspended in LC solvent A ( $1 \%$ acetonitrile, $0.1 \%$ formic acid (FA)) and spiked with iRT kit calibration peptides (Biognosys). Peptide concentrations were determined using a UV/VIS Spectrometer at 280nm (SPECTROstar Nano, BMG Labtech).

\section{HRM (DIA) mass spectrometry acquisition}

For DIA LC-MS/MS measurements, $1 \mu \mathrm{g}$ of peptides per sample were injected to an in-house packed reversed phase column (PicoFrit emitter with $75 \mu \mathrm{m}$ inner diameter, $60 \mathrm{~cm}$ length and $10 \mu \mathrm{m}$ tip from New Objective, packed with $1.7 \mu \mathrm{m}$ Charged Surface Hybrid C18 particles from Waters) on a Thermo Scientific ${ }^{\text {TM }}$ EASY-nLC ${ }^{\text {TM }} 1200$ nano-liquid chromatography system connected to a Thermo Scientific ${ }^{\text {TM }}$ Orbitrap Fusion $^{\text {TM }}$ Lumos $^{\text {TM }}$ Tribrid $^{\text {TM }}$ mass spectrometer equipped with a Nanospray Flex ${ }^{\text {TM }}$ Ion Source. LC solvents were A: $1 \%$ acetonitrile in water with $0.1 \%$ FA; B: $20 \%$ water in acetonitrile with $0.1 \%$ FA. The nonlinear LC gradient was $1-59 \%$ solvent $B$ in 95 minutes followed by $59-90 \%$ B in 10 seconds, $90 \%$ B for 8 minutes, $90-1 \% \mathrm{~B}$ in 10 seconds and $1 \% \mathrm{~B}$ for 5 minutes at $60^{\circ} \mathrm{C}$ and a flow rate of $250 \mathrm{nl} / \mathrm{min}$. The DIA method consisted of one full range MS1 scan and 29 DIA segments was adopted from Bruderer et al. ${ }^{40}$.

\section{HRM data analysis}

HRM data were first analyzed using a directDIA search using Biognosys' search engine SpectroMine, the false discovery rate on peptide and protein level was set to $1 \%$. A human UniProt fasta database (Homo sapiens, 2020-07-01) was used for the search engine, allowing for 2 missed cleavages and variable modifications ( $\mathrm{N}$-term acetylation, methionine oxidation). HRM mass spectrometric data were analyzed using Spectronaut ${ }^{\mathrm{TM}} 14$ software (Biognosys). The false discovery rate on peptide and protein level was set to $1 \%$, data was filtered using row-based extraction. The assay library (protein inventory) generated in this project was used for the analysis. The HRM measurements analyzed with Spectronaut were normalized using local regression normalization ${ }^{41}$; separate normalization was performed for 3 control samples. 
For testing of differential protein abundance, protein intensities for each protein were analyzed using a two-sample sample Student's t-test. The following thresholds were applied for candidate identification: pvalue $<0.05$; absolute average log2 ratio > 0.58 (fold-change $>1.5$ ). Principal component analysis was conducted in $\mathrm{R}$ using prcomp and a modified ggbiplot function for plotting, and partial least squares discriminant analysis (PLS-DA) was performed using the mixOMICS package. PLS-DA model fitting was performed on the samples from stimulated cells. Functional analysis was performed using String-db (string-db.org, version 11). General plotting was done in R using the ggplot2 package.

\section{Neurite Outgrowth Assay}

96 well plate was coated with $50 \mu \mathrm{l} /$ well $1 \%$ Matrigel in DMEM at $4^{\circ} \mathrm{C}$ overnight. PC12 cells were plated into coated plate at a density of $3 \times 10^{3}$ cells/well in $100 \mu$ growth medium and cultured for $24 \mathrm{~h}$. Cells were incubated with $200 \mu \mathrm{l} /$ well of fresh medium containing test compounds for 7 days followed by fresh medium replacing every 3 days. Cells were fixed with $4 \%$ paraformaldehyde and incubated for 20 min at room temperature (RT), then they were rinsed in PBS and incubated with permeabilization buffer (0.1\% Triton-X 100 in PBS) for 20 min at RT. Followed PBS washing, cells were incubated with $\beta 3$-Tubulin primary antibody $(1: 1000)$ in antibody buffer ( $3 \%$ BSA in PBS $+0.05 \%$ Tween) at $4{ }^{\circ} \mathrm{C}$ overnight. Cells were rinsed with PBST (PBS $+0.05 \%$ Tween) and incubated with secondary antibody $(1: 1000)$ and Hoechst (1:1000) in antibody buffer for $2 \mathrm{~h}$ at RT away from light. After PBS washing, cells images were taken by the High-Content Imaging System, CellInsight CX5 (ThermoFisher Scientific). The nuclei were stained by Hoechst and the neuron cell bodies and neurites were stained with secondary antibodies conjugated Alexa Fluor 488. 10x objective was used and 9 fields/well were captured in both nuclei and neurite channels. The average cell number and neurite length per field were measured by the neurite analysis module of HCS.

\section{Human iPSC-derived Microglial Phagocytosis Assay of Amyloid $\beta-42$}

Human iPSC-induced microglia (BrainXell, BX-0900) were cultured in microglia culture medium for 5 days according to the instruction. FITC-labeled $A \beta 42$ proteins were dissolved with $2 \mathrm{mM} \mathrm{NaOH}$ and diluted with PBS to $30 \mu \mathrm{M}$ followed by aging in the dark at $37^{\circ} \mathrm{C}$ for $2 \mathrm{hr}$ to form aggregated FITC-A $\beta 42$. The microglia were treated with compounds at different concentrations for $30 \mathrm{~min}$ followed by addition of aggregated FITC-A 42 with final concentration of $0.3 \mu \mathrm{M}$. After incubation for $24 \mathrm{hr}$, the microglia were fixed with $4 \%$ PFA at RT for $20 \mathrm{~min}$ and then subjected to staining solution containing $1 \mu \mathrm{g} / \mathrm{mL}$ Hoechst-33342 and 100

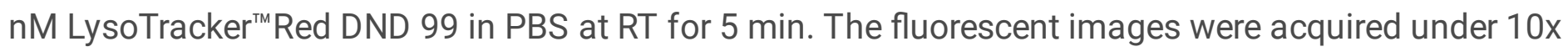
objective using PE Operetta CLS System and the positive cell percentage with phagocytosed FITC-Aß42 was quantified.

\section{Detection of Secreted Proteins using the MSD Assay Platform}


HMC3 cells were resuspended, counted using the LUNA-II Automated Cell Counter, seeded in the 6-well plate ( $400 \mathrm{~K}$ cells $/ 2 \mathrm{ml}$ medium/well), and incubated overnight to allow cells to attach. The media and detached cells were removed next day. The cell layer was washed two times in PBS and once in serumand phenolred-free DMEM (SPFM). Cells were incubated in SPFM for $4 \mathrm{hr}$ prior to treatment with TNF-a $(0.3 \mu \mathrm{g} / \mathrm{ml})$ and/or Cromolyn $(0.3 \mu \mathrm{M}, 3 \mu \mathrm{M}, 10 \mu \mathrm{M}, 30 \mu \mathrm{M})$ or F-cromolyn-diacid $(0.3 \mu \mathrm{M}, 3 \mu \mathrm{M}, 10 \mu \mathrm{M}, 30$ $\mu \mathrm{M})$ for $24 \mathrm{hr}$. The conditioned medium was collected and centrifuged at 1,000 x RCF (g) for 5 min to pellet detached cells and large debris, which was subsequently passed through a $0.22 \mu \mathrm{m}$ filter with PVDF membrane to remove smaller debris. Samples of the supernatant medium were put in a CoolRack (\#07210041, Fisher Scientific) on dry ice for Snap-freezing and kept at $-80^{\circ} \mathrm{C}$ until use.

Meso-scale V-PLEX plates that detect a panel of human cytokines including IL-2, IL-4, VEGF-A, and a panel of human chemokines including GRO-a/CXCL1, Eotaxin/CCL11, were used as per manufacture's protocol. $25 \mu \mathrm{L}$ of the conditioned medium was used in each well of the MSD plates to detect the analytes. The plates were then analyzed on an MSD QuickPlex SQ120 instrument.

\section{PI3K-ס Lipid Kinase Activity via ADP-Glo Enzyme Assay}

Activity-based PI3K- $\delta$ (p110d/p85a) lipid kinase assay kit for compound testing via ADP-Glo kinase system was used according to the manufacture's protocol (Reaction Biology). PI3K- $\delta$ enzyme was diluted in prepared PI3K- $\delta$ Reaction Buffer/Lipid Substrate mixture. $0.5 \mu \mathrm{L}$ of testing compound solution, $4 \mu \mathrm{L}$ of enzyme/lipid mixture, and $0.5 \mu \mathrm{L}$ of $250 \mu \mathrm{M}$ ATP in water were added to the wells of a 384-well low volume plate and incubated at RT for $60 \mathrm{~min} .5 \mu \mathrm{L}$ of ADP-Glo Reagent with $10 \mathrm{mM} \mathrm{MgCl}_{2}$ were added to each well and incubated at RT for 40 min, followed by addition of $10 \mu \mathrm{L}$ Kinase Detection Reagent and incubation at RT for another $30 \mathrm{~min}$. Luminescence was recorded as the readout for kinase activity.

\section{Quantification and Statistical Analysis}

All the data were presented as mean \pm standard error from at least three times, each done in triplicate. The statistical significance between two groups was determined by Student's $t$ test, whereas the comparisons of multiple groups were carried out by one-way ANOVA, followed by Bonferroni's post-test using GraphPad Prism 7 (GraphPad Software, Inc.). A probability value of * $p<0.05$ was considered to be significant.

\section{Results}

\section{TNF- $a$ is a robust inducer of differentially expressed genes and proteins in HMC3 human microglia}

RNA-seq analysis found the response by HMC3 microglia to $0.3 \mu \mathrm{g} / \mathrm{mL}$ TNF-a resulted in differential expression of 1061 genes out of 26,485 genes quantified (Fig. 1a). Interestingly, Function-to-Gene Association Network (FGNet) analysis revealed TNF-a affects many cellular functions including organization and degradation of the extracellular matrix (ECM), which have been associated with fibrotic 
diseases ${ }^{42}$, as well as collagen degradation (Fig. 1b). Many genes were differentially altered including MMP1, MMP3, MMP9, CXCL2, CXCL5, CXCL8, CXCL10, IL-6, COL21A1, COL12A1, COL1A2, and TGFB2

(Fig. 1b and Supplementary Figure S1a). This observation is further highlighted by a pathway enrichment dotplot in Fig. 1c, showing that 40 or more genes significantly changed by TNF- $a$ are involved in ECM organization and interleukin signaling, respectively; 20 or more genes each associated with degradation or formation of collagen and collagen fibril assembly, respectively; and 20 or more genes involved with ECM proteoglycans. The extent of interconnected cellular networks of fibrosis affected by TNF-a treatment of HMC3 microglia is further shown in Supplementary Figure S1b, with an enriched pathway network plot showing interconnections of ECM organization, collagen biosynthesis/assembly/formation, and ECM degradation. HRM-ID + mass spectrometry was used for the unbiased quantification of all detectable peptides and proteins, which resulted in quantification of 3,730 proteins, 42,435 peptides and 59,076 peptide ion variants across all samples tested. Out of these, a total of 3,427 proteins were changed significantly. String-db functional analysis of Protein-Protein Interaction Network (PPINet) incorporating known and predicted interactions shows a complex, dense network of connections between significantly-altered proteins in HMC3 microglia due to TNF-a (Fig. 1d and Supplementary Figure S1c). There is an extensive number of edges that interconnect between many proteins related to stress response (green sphere), cytokine signaling (blue spheres), response to cytokines (red spheres), and chemokine signaling (yellow spheres). The dense network of strings in the PPINet analysis indicates that TNF-a elicits a robust response in HMC3 microglia that likely affects more biologically-connected proteins than a random assortment of proteins would ${ }^{43}$.

\section{Cromolyn and F-cromolyn suppress TNF-a-induced fibrosis and inflammation in HMC3 microglia}

As TNF-a was found to exhibit a pro-fibrotic and pro-inflammatory profile for HMC3 microglia in our experiments, we next sought to determine cromolyn's ability to influence this process. RNA-sequencing analysis found that adding $30 \mu \mathrm{M}$ cromolyn to $0.3 \mu \mathrm{g} / \mathrm{mL}$ TNF-a induced HMC3 microglia differentially regulated the expression of 1441 genes with a cutoff $p$-value $<0.05$ (Fig. 2a). FGNet analysis revealed that $30 \mu \mathrm{M}$ cromolyn significantly affects cellular pathways associated with ECM organization, degradation of ECM, assembly of collagen fibrils and collagen degradation (Fig. 2b; Supplementary Figures S2a and S2b).

As a majority of the genes altered by cromolyn affect ECM dynamics, we next performed proteomics analyses using HRM-ID + mass spectrometry of the cell supernatant of TNF-a-treated HMC3 microglial cells in the presence of cromolyn or F-cromolyn (Fig. 2c). We found that TNF-a induced secretion of collagen XVIII alpha-1 (COL18A1) relative to control $(p=0.0009)$ and $0.3 \mu \mathrm{M}$ and $3 \mu \mathrm{M}$ cromolyn treatment achieved similar decreases in COL18A1 secretion ( $p=0.0094$ and $p=0.005$, respectively) (Fig. 2c). $0.3 \mu \mathrm{M}$ F-cromolyn significantly decreases COL18A1 further $(p=0.0003)$ than either concentration of cromolyn relative to TNF- $a$ treatment alone and $3 \mu \mathrm{M}$ F-cromolyn decreases COL18A1 secretion further still $(p<0.0001$ ) (Fig. $2 c)$. We also found that addition of $0.3 \mu \mathrm{M}$ and $3 \mu \mathrm{M}$ cromolyn 
after TNF-a treatment to HMC3 microglia mildly reduced fibronectin secretion but reduction of fibronectin caused by $0.3 \mu \mathrm{M}$ and $3 \mu \mathrm{M}$ F-cromolyn addition was more significantly pronounced $(p=0.0004$ and $p=$ 0.0005 , respectively) (Fig. 2c). Additionally, we observed that ECM component Tenascin-c (TNC) secretion was strongly upregulated by $0.3 \mu \mathrm{g} / \mathrm{mL}$ TNF-a $(p<0.0001)$ (Fig. $2 \mathrm{c})$. Though addition of $0.3 \mu \mathrm{M}$ or $3 \mu \mathrm{M}$ cromolyn modestly reduced TNC secretion, $0.3 \mu \mathrm{M}$ and $3 \mu \mathrm{M}$ F-cromolyn significantly reduced TNC secretion by TNF- $a$ induced HMC3 microglia ( $p=0.0011$ and $p=0.0003$, respectively) (Fig. $2 c)$. Interestingly, only cromolyn $(0.3 \mu \mathrm{M}$ and $3 \mu \mathrm{M})$ significantly reduced secretion of the keratin proteins KRT9 $(p<0.0001$ and $p<0.0001)$, KRT1 $(p=0.0034$ and $p=0.0002)$ and KRT5 $(p=0.0095$ and $p<0.0001)$ post TNF-a treatment (Fig. 2c). Figure 2d shows a 3D principal component analysis (PCA) of the collective gene expression of HMC3 microglia controls relative to all treatment groups. More separation between clusters of points represents more dissimilar gene expression profiles. $30 \mu \mathrm{M}$ cromolyn (green cluster) and $30 \mu \mathrm{M}$ F-cromolyn (grey cluster) groups separate from the TNF-a group (yellow cluster) in the PCA plot from the control group (blue cluster). This highlights that across five independent experiments, cromolyn and F-cromolyn differentially alter the collective gene expression of TNF-a induced HMC3 microglial cells.

Next, we determined through RNA-seq analysis that $30 \mu \mathrm{M}$ F-cromolyn administration to $0.3 \mu \mathrm{g} / \mathrm{mL}$ TNF-ainduced HMC3 microglia differentially expressed 2108 genes using a cutoff p-value $<0.05$ (Fig. 3a). Performing an FGNet analysis confirmed that $30 \mu \mathrm{M}$ F-cromolyn also affects fibrosis and inflammationrelated cellular pathways, including collagen fibril assembly and neutrophil degranulation (Fig. 3b; Supplementary Figures S3a and S3b). MSD V-Plex cytokine assays for GRO-a, Eotaxin, VEGF-A, IL-2, and $\mathrm{IL}-4$ in the presence of $30 \mu \mathrm{M}$ cromolyn and F-cromolyn are provided in Fig. 3c and Fig. 3d. TNF-a potently induced GRO-a secretion in HMC3 microglia $(p<0.0001)$. $30 \mu \mathrm{M}$ cromolyn and $30 \mu \mathrm{M}$ F-cromolyn each significantly reversed GRO- $a$ secretion induced by TNF- $a$ treatment $(p<0.0001$ and $p<0.0001$, respectively) (Fig. 3c and Supplementary Figure S3c). TNF-a treatment also significantly increased Eotaxin expression $(\mathrm{p}<0.0001)$ and administration of either $30 \mu \mathrm{M}$ cromolyn or $30 \mu \mathrm{M}$ F-cromolyn decreased Eotaxin levels by approximately 40\% ( $p<0.0001$ and $p<0.0001$, respectively) (Fig. 3c). We also observe that $0.3 \mu \mathrm{g} / \mathrm{mL}$ TNF-a treatment increased secretion of VEGF-A in HMC3 microglia ( $\mathrm{c}<$ $0.0001)$, but $30 \mu \mathrm{M}$ cromolyn reduced VEGF-A secretion to pre-TNFa treatment levels $(p<0.0001)$. Additionally, $30 \mu \mathrm{M}$ F-cromolyn reduced VEGF-A secretion after TNF-a treatment $(p<0.0186)$ (Fig. $3 c)$. Although in a previous study ${ }^{19}$ we did not observe significant upregulation of IL-2 or IL-4 in the presence of $0.3 \mu \mathrm{g} / \mathrm{mL}$ TNF-a, we were able to show over many repeat experiments that TNF-a is able to significantly increase secretion of both cytokines (Fig. 3c and 3d). IL-2 secretion by HMC3 microglia is promoted following $0.3 \mu \mathrm{g} / \mathrm{mL}$ TNF-a administration $(p<0.0001)$, but is significantly decreased following $30 \mu \mathrm{M}$ cromolyn or $30 \mu \mathrm{M}$ F-cromolyn treatment ( $p<0.0001$ and $p<0.0001$, respectively) (Fig. $3 \mathrm{c}$ ). $30 \mu \mathrm{M}$ cromolyn and F-cromolyn significantly increased IL-4 secretion after $0.3 \mu \mathrm{g} / \mathrm{mL}$ TNF-a treatment $(\mathrm{p}<$ 0.0001 and $p<0.0001$, respectively) (Fig. $3 d$ ). This finding is important as IL-2 is a pro-inflammatory cytokine with prominent roles in T-cell proliferation and differentiation ${ }^{44}$ and IL-4 is an anti-inflammatory cytokine capable of suppressing NF-KB signaling in monocytes to inhibit secretion of pro-inflammatory cytokines IL-6 and IL- $1 \beta^{45}$. 
HRM-ID + mass spectrometric proteomic assays determined that TNF-a increases PLP1 secretion by HMC3 microglia (Fig. 3e). Although $0.3 \mu \mathrm{M}$ cromolyn did not significantly change PLP1 secretion, $3 \mu \mathrm{M}$ cromolyn drastically decreases average PLP1 secretion below control levels $(p<0.0001)$. Both $0.3 \mu \mathrm{M}$ and $3 \mu \mathrm{M}$ F-cromolyn also decrease PLP1 secretion relative to TNF-a treatment alone $(p<0.0001$ and $p<$ 0.0001), though there is no apparent difference in inhibition based on concentration. We also observed increased secretion of PELP1 after $0.3 \mu \mathrm{g} / \mathrm{mL}$ TNF-a treatment. $0.3 \mu \mathrm{M}$ cromolyn modestly decreases PELP1 secretion induced by TNF-a, but increasing concentration to $3 \mu \mathrm{M}$ cromolyn significantly reduces PELP1 levels $(p<0.0001)$. Repeating the experiment with $0.3 \mu \mathrm{M}$ and $3 \mu \mathrm{M}$ F-cromolyn achieved similar decreases in PELP1 secretion ( $p=0.0049$ and $p<0.0001)$ as to what was observed with respective concentrations of cromolyn (Fig. 3e). We also found that TNF-a administration strongly induces HSP90 secretion in HMC3 microglia $(p<0.0001)$. Subsequent treatment with either $0.3 \mu \mathrm{M}$ or $3 \mu \mathrm{M}$ concentrations of cromolyn or F-cromolyn resulted in a significant decrease in HSP90 secretion after TNF-a administration ( $p<0.0001$, respectively).

\section{Cromolyn and F-cromolyn augment NGF-induced neurite outgrowth in PC12 neuronal cells}

As reduction in neuron processes is characteristic of neurodegeneration ${ }^{46}$, we sought to determine the effect of cromolyn and F-cromolyn on the neurite outgrowth of PC12 neuronal cells. Figure 4a shows PC12 cells maintain similar morphology when exposed to $100 \mu \mathrm{M}$ cromolyn in comparison to vehicle, suggesting that cromolyn alone does not have measurable effect on neurite outgrowth. As expected, treating PC12 cells with $7.5 \mathrm{ng} / \mathrm{mL}$ nerve growth factor (NGF) (see Supplementary Figure S4 for NGF concentration optimization) results in noticeable extension of neurites $(p<0.0001)$ (Fig. 4b). Although concomitant addition of $30 \mu \mathrm{M}$ cromolyn with NGF does not appear to change PC12 cell morphology, increasing the concentration of cromolyn to $100 \mu \mathrm{M}$ results in significant extension of neurite length $(\mathrm{p}=$ 0.0032) (Fig. 4b). We also observe decreases in cell numbers with treatment of NGF (Fig. 4b), which is known to be due to the anti-proliferative signaling of $\mathrm{NGF}^{47}$ and not due to any cytotoxicity of cromolyn. Under NGF treatment, PC12 cells were differentiated into neuron-like cells that cannot proliferate, so the cell number of NGF addition groups were less than vehicle group. As we observe similar results in neurite outgrowth for identical experiments with F-cromolyn (Fig. 4c). Altogether, these data indicate that higher concentrations of cromolyn and F-cromolyn augment the capability of NGF to promote neurite outgrowth in PC12 neuronal cells.

HRM-ID + mass spectrometric proteomic assays determined that TNF-a increases tubulin tyrosine ligase (TTL) secretion by HMC3 microglia ( $p=0.0024)$ (Fig. $4 \mathrm{~d}$ ). Addition of $0.3 \mu \mathrm{M}$ and $3 \mu \mathrm{M}$ cromolyn significantly increased TTL secretion after $0.3 \mu \mathrm{g} / \mathrm{mL}$ TNF-a exposure $(p=0.0088$ and $p=0.0015$, respectively). F-cromolyn is more effective at increasing TTL secretion than cromolyn is, as $0.3 \mu \mathrm{M} \mathrm{F-}$ cromolyn ( $p=0.0014)$ achieves similar TTL levels to that of $3 \mu \mathrm{M}$ cromolyn. Increasing to $3 \mu \mathrm{M} \mathrm{F-}$ cromolyn continues this trend and results in the highest TTL level observed in our experiments, relative to TNF- $a$ administration alone $(p<0.0001)$. We also observed increased PROX1 secretion after addition of 
TNF-a $(p=0.0784)$ and significantly higher PROX1 levels in concert with $0.3 \mu \mathrm{M}$ cromolyn $(p=0.0033)$ (Fig. 4d). $3 \mu \mathrm{M}$ cromolyn slightly decreased PROX1 levels relative to $0.3 \mu \mathrm{M}$ cromolyn, but still achieved significantly higher secretion of PROX1 relative to TNF- $a$ alone $(p=0.0003)$. Both $0.3 \mu \mathrm{M}$ and $3 \mu \mathrm{M}$ Fcromolyn achieved similar increases in PROX1 secretion relative to TNF- $\alpha$ alone $(p=0.0005$ and $p=$ 0.0002 , respectively). Further, we observed significant increase in Rab35 secretion after administering TNF-a to HMC3 microglia ( $p=0.0001)$ (Fig. 4d). Although only a slight increase in secretion was noted for $0.3 \mu \mathrm{M}$ cromolyn, $3 \mu \mathrm{M}$ cromolyn increased Rab35 secretion significantly relative to TNF-a alone $(p=$ 0.0002). $0.3 \mu \mathrm{M}$ F-cromolyn treatment resulted in modest increase of Rab35 secretion relative to TNF-a alone, but the higher $3 \mu \mathrm{M}$ F-cromolyn treatment had significant increase $(p=0.0074)$. Lastly, we noted a strong increase in CSDE1 secretion upon $0.3 \mu \mathrm{g} / \mathrm{mL}$ TNF-a treatment $(p<0.0001)$ (Fig. 4d). Addition of $0.3 \mu \mathrm{M}$ or $3 \mu \mathrm{M}$ cromolyn achieved similar decreases in CSDE1 relative to TNF- $a$ alone $(p=0.0016$ and $p$ $=0.0003$ ). Interestingly, F-cromolyn treatment at both $0.3 \mu \mathrm{M}$ and $3 \mu \mathrm{M}$ achieved similar significant decreases in CSDE1 relative to TNF- $a(p<0.0001$ and $p<0.0001)$, but both resulted in lower CSDE1 secretion levels than either concentration of cromolyn.

Cromolyn and F-cromolyn promote iPSC-derived human microglial phagocytosis of Amyloid $\beta$-protein 42 (Aß42) via PI3K signaling

Microglia are the resident macrophages of the CNS and the promotion of their phagocytic profile is important to limit the extent of inflammatory activation that can lead to neurodegeneration. This is particularly important as aggregate, extracellular amyloid $\beta$-protein is largely associated with neuron toxicity. PI3K signaling is regulated by histone modifications ${ }^{48}$ and deletion of histone deacetylases 1 and 2 have led to increased microglial phagocytosis of amyloid plaques in FAD DKO mice models ${ }^{49}$. We found through RNA-seq analysis that both $30 \mu \mathrm{M}$ cromolyn and $30 \mu \mathrm{M}$ F-cromolyn significantly decreased PIK3CD mRNA levels in HMC3 microglia ( $p=0.0065$ and $p=0.0002$, respectively) (Fig. 5a). In light of this finding, we then confirmed via PI3K- $\delta$ lipid kinase assay that cromolyn dose-dependently reduced PI3K- $\delta$ enzyme activity (Fig. 5b). We then utilized iPSC-derived human microglia to ascertain the ability of cromolyn and F-cromolyn to promote microglia phagocytosis. Firstly, we determined the microglial purity of these iPSC-differentiated microglial cells to be more than $95 \%$ using IF staining for IBA-1, which is considered a marker of microglia (Supplementary Figure S5a). Secondly, we determined to use the microglial cell number of 2,000 cell/well and the FITC-labeled A 342 concentration of $0.3 \mu \mathrm{M}$ to conduct compound evaluation (Supplementary Figure S5b). In determining the concentration to use for the promotion by cromolyn and F-cromolyn to promote microglia phagocytosis, we compared increasing concentrations of each compound to the quantitative phagocytosis (POS) ratio; higher POS ratio corresponds to more $A \beta$ internalization compared to all cells in the image (Fig. $5 \mathrm{c}$ ). We found that $1 \mathrm{mM}$ treatment for both cromolyn and F-cromolyn resulted in the most significant change in POS ratio relative to $0.2 \%$ DMSO control $(p<0.0001$ and $p=0.0001$, respectively) and was thus used to show cromolyn and F-cromolyn's modulatory effects on microglial phagocytosis. The left panel of Fig. $5 \mathrm{~d}$ shows the FITC$A \beta 42$ image of control iPSC microglia. FITC-A $\beta$ is distributed fairly evenly throughout the image with few spots of increased localization observed. Comparing both the left panel of Fig. $5 \mathrm{~d}$ to the right image 
overlay of Hoechst stain for nuclei (blue) and lysotracker for cell lysosomes (orange), it becomes apparent that clumping of FITC-A $\beta$ resides within the lysosomes of the microglia. Upon addition of $1 \mathrm{mM}$ cromolyn to iPSC-derived microglia, the fluorescence of FITC-A $\beta$ is brighter and more concentrated in specific areas of the image (Fig. 5e, left). Comparing to the right overlay image (Fig. 5e, right), it is clear that microglia have upregulated their phagocytosis of FITC-A $\beta$ by the intense colocalization of $A \beta$ (green) within the lysosome (orange). Because the change in POS ratio for $1 \mathrm{mM}$ cromolyn and $1 \mathrm{mM}$ F-cromolyn are very similar, we only show representative images for $1 \mathrm{mM}$ cromolyn.

\section{Discussion}

Attenuating inflammatory signaling is a preemptive strategy to avoid downstream neurodegenerative processes such as inflammation-induced neuronal damage and fibrosis. One such route is the $\mathrm{PI} 3 \mathrm{~K} / \mathrm{Akt} / \mathrm{mTOR}$ inflammatory signaling pathway ${ }^{44}$. We have previously shown that TNF-a treatment to HMC3 microglia induces a pro-inflammatory phenotype and that cromolyn and F-cromolyn are able to significantly reduce secretion of an array of important cytokines and chemokines, including IL-1 $\beta$, IL-6, IL8, IFN- $y$, CCL2, CCL3, CCL4, and CXCL10 19. IL-2 is another pro-inflammatory cytokine that can activate NF-KB to promote expression of IL- 6 and MCP-1, and disrupt the integrity of the blood-brain barrier by comprising components of the adherens junctions ${ }^{50}$. In contrast, IL-4 has been associated with protective mechanisms of brain ischemia and can induce expression of anti-inflammatory M2 microglial phenotype in primary rat cortical glia cultures, and encourage PPARy-dependent microglial phagocytosis ${ }^{51}$. Increased expression of GRO- $a$ (also known as CXCL1) in monocytes from APP/PS1 transgenic mice and $A D$ patients have been implicated in increased monocyte infiltration into the brain ${ }^{52}$ as well as massive neutrophil recruitment in other models of brain and tissue damage ${ }^{53,54}$. The inflammatory cytokine Eotaxin is found in many cell types, including microglia and astrocytes, with roles in neurogenesis suppression and is expressed in heightened levels in aged human plasma and CSF ${ }^{55}$. Additionally, increased levels of VEGF have also been found in CSF and serum of AD patients relative to controls ${ }^{56,57}$. LPS-activated microglia from SD rats have been shown to upregulate VEGF-A expression, increase angiogenesis and promote the migration and proliferation of retinal microvascular endothelial cells while impairing tight junction protein expression ${ }^{58}$. In the context of prolonged inflammation, weakening of the BBB and increasing proliferating cells into the brain that respond to inflammatory stimuli could allow for increased microglial inflammatory activation. In this report, we further show that cromolyn and Fcromolyn promote suppression of TNF-a induced secretion of the three pro-inflammatory mediators IL-2, GRO-a, Eotaxin, and VEGF-A, and are able to increase the expression of anti-inflammatory IL-4. This finding indicates that cromolyn and F-cromolyn are able to modulate key mediators of neuroinflammation in $\mathrm{HMC} 3$ microglia that are related to downstream neurodegenerative disease progression.

Unbiased proteomic profiling of HMC3 microglia found that cromolyn and F-cromolyn also modulate other inflammatory proteins. Proteolipid protein 1 (PLP1) is the major form of myelin protein in the CNS with roles in myelin sheath structure, oligodendrocyte proliferation, and axonal survival ${ }^{59}$. Plp1tg mouse 
models exhibited heightened levels of PLP1 and significant microglial inflammation activation throughout brain parenchyma before myelinated fibers were present, leading to 24-fold TNF- $\alpha$ and 7 -fold IL-6 increases compared to wildtype mice ${ }^{60}$. Therefore, decreases in PLP1 gene expression due to cromolyn (-14.62 fold change decrease after TNF-a exposure, Supplementary Figure S2a) may be responsible, in part, for its anti-inflammatory capabilities. We also found that cromolyn and F-cromolyn decrease Proline, Glutamate And Leucine Rich Protein 1 (PELP1) secretion following TNF-a administration. PELP1 is widely expressed in the brain, particularly in the cell membrane, dendritic shafts and synaptic terminals of neurons in the forebrain ${ }^{61}$. PELP1 has been associated with pro-survival mechanisms after ischemic injury by activating ERK and Akt pathways, as well as E2 inhibition of GSK3 3 , a key mediator of cell death in $A D^{61}$. PELP1 can promote NF-KB gene expression to activate macrophages ${ }^{62}$. PELP1 knockdown in medulloblastoma cells results in downregulation of NF-KB, including TRAF1 and IL-8, as well as expression ECM-related genes MMP7, MMP9, and MMP14 ${ }^{63}$, the latter of which is upregulated by microglia in late-stage NDD development ${ }^{64}$. Interestingly, PELP1 is a substrate of GSK3 $\beta$, interacts with PI3K, and its expression may be determined by the E2-ER-GSK3 $\beta$ signaling loop ${ }^{65}$. Dose-dependent decreases in PELP1 secretion by both cromolyn and F-cromolyn may contribute to the ameliorating anti-inflammatory activation of HMC3 microglia observed in our experiments through modulation of downstream PI3K, GSK3 $\beta$, and NF-KB signaling pathways.

The heat shock protein HSP90 is a diverse chaperone protein with regulatory roles in GSK-3 $\beta$ stabilization and tau phosphorylation ${ }^{66}$; as well as downstream phosphorylation of Akt and PI3K/Akt/mTOR signaling ${ }^{67,68}$. HSP90 is also associated with pro-inflammatory release of cytokines such as IL-6, IL-1 $\beta$, and TNF- $a$, and the inhibition of the constitutive cytoplasmic HSP90 $\beta$ isoform ${ }^{69}$ decreases proinflammatory cytokine signaling, ERK phosphorylation, and STAT3 in the cytosol in N9 microglia ${ }^{70}$. Studies with TNF $\Delta^{\mathrm{ARE}}$ mice with high circulating levels of TNF-a have shown that inhibition of the Cterminal ATPase of HSP90 with novobiocin prevents Akt stabilization, increasing its degradation, and subsequent decrease in production of TNF- $\mathrm{a}^{71}$. Others found that HSP90 $\beta$ inhibition by novobiocin in the Hs578T cell line lowers soluble and insoluble fibronectin levels suggesting that, in addition to HSP90 $\beta$ binding directly to fibronectin, HSP90 $\beta$ is also involved in ECM assembly ${ }^{72}$. Interestingly, affinity chromatography was previously utilized to find that cromolyn binds to the N-terminus of wildtype HSP9O to inhibit its $\mathrm{N}$-terminal chaperone capabilities, but not to its $\mathrm{C}$-terminus ${ }^{73}$. In our experiments, treatment with cromolyn and F-cromolyn results in significant decrease in HSP90 after addition of TNF-a. The modulation of HSP90 secretion by HMC3 microglia may be partly responsible for the upstream antiinflammatory capabilities of cromolyn and F-cromolyn, in addition to downstream PI3K/Akt/mTOR and neurodegenerative GSK-3 $\beta$ signaling.

Collagens are the main structural proteins of the extracellular matrix and there are many isoforms located within the body. Expression of collagen IV, a major component of the basal laminae, and fibronectin was found to be increased in frontal and temporal cortex cerebral microvessels in early Alzheimer's Disease and was positively correlated with amyloid deposition compared to controls ${ }^{26}$. Collagen XVIII associates 
with vascular deposits of amyloid beta and senile plaques, but not tau, and is deposited at higher rates in the $\mathrm{AD}$ brain compared to age-matched controls ${ }^{74}$. Interestingly, the $\mathrm{C}$-terminal fragment of collagen XVIII is endostatin, an anti-angiogenic protein ${ }^{75}$. Endostatin has strong associations with neurological disease and can dose-dependently bind and sequester nerve growth factor (NGF) to prevent neurite outgrowth and migration in PC12 cells ${ }^{75}$. Increased collagen XVIII secretion has also been associated with increased VEGF expression in experiments with hepatocellular carcinoma ${ }^{76}$. We also observe increased concomitant secretion of collagen XVIII and VEGF (Fig. 2c and Fig. 3c, respectively), but also subsequent decreases in both proteins after cromolyn and F-cromolyn treatment.

Collagen production and fibrosis progression are intimately linked and PI3K/Akt signaling has been associated with both ${ }^{77-79}$. COL6A3 has been implicated as the main isoform of collagen $\mathrm{VI}$ that promotes downstream pro-fibrotic regulation of myofibroblasts ${ }^{80}$. COL6A3 depletion results in reduced migratory ability of myofibroblasts and reduced recruitment of THP-1 myeloid cells to myofibroblastconditioned media ${ }^{80}$. We observed significant downregulation of COL6A3 gene expression by TNF-a induced HMC3 microglia by $30 \mu \mathrm{M}$ cromolyn and $30 \mu \mathrm{M}$ F-cromolyn (-2.46 and -2.03 fold change from TNF-a treatment alone, respectively) (Supplementary Figure S2a and S3a). Additionally, gene expression of other collagens significantly decreased by cromolyn after TNF- $a$ administration include COL6A3, COL1A2, COL9A3, and COL8A1 (Supplementary Figure S2a). F-cromolyn also significantly reduced gene expression of COL15A1, COL6A3, COL7A1, COL9A3, COL16A1, and COL8A1 relative to treatment with TNF-a alone (Supplementary Figure S3a). Chronic immobilization stress of rats found extensive remodeling of the hippocampus with increased ECM production and reduced ECM degradation, linking the increased capacity of collagen synthesis with chronic inflammation and immunological dysregulation of the hippocampus ${ }^{81}$. It is possible that downregulation of collagen gene expression and secretion by cromolyn and F-cromolyn in HMC3 microglia in the presence of pro-inflammatory TNF-a affects Akt/PI3K signaling and may attenuate microglia-mediated fibrotic mechanisms initiated during chronic neuroinflammation.

Fibronectin is another essential glycoprotein component of the extracellular matrix in all tissues of the body and its deposition is upregulated during fibrosis ${ }^{82}$. Fibronectin functions in a regulatory role for healthy deposition of ECM as use as a structural scaffold, in addition to modulating cell function during tissue repair ${ }^{82}$. This function continues into the CNS, as fibronectin binds collagens, proteoglycans, and other ECM proteins to reinforce the endothelial basement membrane of the $\mathrm{BBB}^{83}$. Aberrant amounts of aggregate fibronectin deposition, as seen with multiple sclerosis (MS), lead to abnormal ECM structure and impaired remyelination ${ }^{84}$. Normally these aggregates activate cellular repair mechanisms that then activate microglia and macrophages to a phagocytic phenotype, but in the presence of IFN-y it was shown to perpetuate inflammation, particularly by increased macrophage and microglia integrindependent production of nitric oxides ${ }^{84}$. Interestingly, cromolyn has been previously shown to inhibit mast cell-derived histamine to decrease hepatic fibrosis in $\mathrm{Mdr}^{-/-}$mice ${ }^{85}$. Inhibition of mast cell-derived histamine with administration of cromolyn decreases hepatic fibrosis in $\mathrm{Mdr}^{-/-} \mathrm{mice}^{85}$. Mdr2 ${ }^{-/-}$mice 
have significantly higher expression of fibronectin, collagen type-1a, and a-SMA than wildtype mice and cromolyn administration significantly reduced expression of all three ${ }^{85}$. Further experiments were pursued with cholestatic rats and cromolyn decreased expression of collagen type-1a, fibronectin, and a-SMA in cultured HSCs treated with mast cell supernatants from BDL rats ${ }^{86}$. We observe in our study that TNF-a induced HMC3 microglia secretion of fibronectin was significantly abated by F-cromolyn treatment, suggesting that inflammation-induced expression of fibronectin and potential abnormalities in ECM structure may be stemmed upstream by F-cromolyn.

Epithelial cytoskeletal proteins such as actin and keratin allow for directed integrin signaling that results in fibronectin ECM assembly ${ }^{87}$, with integrins serving as nucleation points for the cytoskeleton of epithelial cells and ECM proteins of the basement membrane ${ }^{88}$. Intriguingly, keratins 5 and 8 are known to be expressed in the choroid plexus, the region of the brain that produces CSF, forms the CSF-blood barrier, and the only region of the brain with significant amounts of epithelial cells ${ }^{89}$. Keratin-1 (KRT1) is found to be excreted in extracellular vesicles by BV2 microglia ${ }^{90}$. It currently remains unclear to what extent these keratins play in the CNS or in neurodegenerative disease, but keratin-9 (KRT9) has been proposed to be a diagnostic biomarker of $A D$ as it has been found exclusively in $A D$ patients' CSF compared to controls ${ }^{91}$. In our experiments, it is interesting that control HMC3 microglia passively secrete KRT1, KRT5, and KRT9 and that keratin secretion is not significantly affected by TNF-a-induced inflammation (Fig. 2c). We observe that only $3 \mu \mathrm{M}$ cromolyn treatment significantly decreases secretion of each keratin below their respective control levels (Fig. 2c) but further studies will be necessary to more fully understand the impact secreted keratin proteins have within the CNS.

Tenascin-c (TNC) is an extracellular matrix glycoprotein that promotes tissue fibrosis in many disease models including liver, lung, MS, and systemic sclerosis; and has strong associations with other inflammatory diseases including cancer, asthma, and Alzheimer's disease ${ }^{92}$. TNC is readily expressed after injury or during inflammation and is important for efficient tissue repair, but has also been associated with pathologic $A \beta$ plaque deposition with concomitant reactive glia ${ }^{93}$. HDAC1 expression is induced by TNC to promote IL- 6 and TNF- $a$ secretion by microglia; these effects were strongly associated with TLR4 signaling, with additional effects on microglia phagocytosis and migration ${ }^{94}$. TNC activation of TLR4 also promotes collagen synthesis and fibroblast activation and is strongly associated with the pathogenesis of systemic sclerosis in mice ${ }^{33}$. In our experiments, TNC secretion was modestly reduced by cromolyn and significantly decreased by F-cromolyn treatment after TNF-a administration to HMC3 microglia. In the context of activation of microglia, others have shown that pretreatment of cromolyn before induced degranulation of brain mast cells reduced TLR4 signaling by microglia and subsequent inhibition of MAPK and Akt inflammatory pathways in Sprague Dawley rats ${ }^{95}$. As TNF-a is able to induce expression of TNC in HepG2 hepatoma cells ${ }^{96}$, it is possible that the reduction of TNF-a-induced TNC secretion by F-cromolyn may also reduce microglia TLR4 and Akt signaling to prevent downstream inflammatory cytokine production and fibrosis-related signaling. 
Tyrosination of microtubules is essential for normal brain development and is readily found in the dendrites and growth cones of neurons ${ }^{97,98}$. a-tubulin in microtubules undergoes a tyrosine removal and addition cycle, the latter of which is catalyzed by tubulin-tyrosine ligase $(T T L)^{99}$. Studies with TTL-null mice found that TTL was essential to maintain timely neurite outgrowth and prevent early axon differentiation, emphasizing that TTL is important for the formal structure and differentiation of microtubules in neurons ${ }^{99}$. This stabilization during microtubule development by TTL may also be important for axon repair post injury, as TTL regulation of tyrosinated a-tubulin has been associated with maintenance of injury signals necessary to activate axon regeneration ${ }^{100}$. Cromolyn and F-cromolyn both induce secretion of TTL by TNF-a activated microglia in our experiments, a factor that may contribute to the stabilization of axonal repair mechanisms during injury and subsequent regeneration of neurites.

Cold shock domain-containing E1 (CSDE1) is an RNA-binding protein that can directly interrupt transcription and translation of proteins and has been shown to prevent neurogenesis in human embryonic stem cells and iPSCs, with dozens of known neurogenesis-related transcripts significantly altered $^{101}$. Gene variants of CSDE1 with heightened number of binding targets, particularly with FMRP, have been associated with autism spectrum disorder ${ }^{102}$. Interestingly, knockdown of CSDE1 in primary mouse cortical neurons resulted in increased neurite and axon length but with fewer and thinner dendritic spines compared to controls, suggesting that CSDE1 is important for successful, morphological outgrowth of neurons ${ }^{102}$. CSDE1 has also been found essential to the formation of stress granules in HeLa cells ${ }^{103}$. Stress granules form within many cell types during extended periods of cellular stress, including oxidative stress, heat shock, TNF-a exposure, and aging, and have been postulated to function as scaffolds for aggregation-prone proteins in Alzheimer's disease and other neurodegenerative disorders ${ }^{104,105}$. Our results confirm that TNF-a administration strongly induces CSDE1 secretion in HMC3 microglia (Fig. 4d). We interpret the significant decreases in CSDE1 secretion following cromolyn and F-cromolyn treatment as having potential to reduce damaging cellular stress responses that may lead to downstream neurodegeneration, as well as to promote neurite outgrowth as observed in coordination with NGF in our PC12 cellular experiments.

Prospero homeobox protein 1 (PROX1) activates NFAT signaling pathway to promote IL-2 transcription in $T$ cells and is associated with terminal differentiation of neural progenitor cells (NPCs) into mature neurons by inhibiting Notch 1 signaling ${ }^{106}$. This pathway is associated with increases in inflammatory gene expression via NF-KB, thereby increasing expression of pro-inflammatory cytokines IL-1 $\beta$, TNF- $\alpha$ and toll-like receptor agonists ${ }^{107}$. Interestingly, oligodendrocyte progenitor NG2 + cells proliferation and differentiation is PROX1-dependent, with PROX1 playing a protective role against destructive glia response of macrophages by upregulating NG2 + cells during the regenerative response to demyelination ${ }^{108}$. PROX1 also plays an important role in suppressing $\mathrm{Ca}^{2+}$ signaling and subsequent neurite outgrowth in mouse and human neuroblastoma cell lines, suggesting that transient PROX1 expression allows newly-formed neurons time to migrate into place before extension of neurites and axons $^{109}$. Although cromolyn and F-cromolyn significantly increase the expression of PROX1 in TNF-a 
induced HMC3 microglia, we do not observe deleterious effects by cromolyn or F-cromolyn in terms of neurite length of PC12 neuronal cells in our experiments. It is possible that cromolyn and F-cromolyn may reduce inflammation by increasing microglial expression of PROX1 to enhance NG2 + cell proliferation in vivo to help curb microglia-related neurodegeneration ${ }^{108}$, but further experiments will be required to confirm if this is an operational mechanism of cromolyn and F-cromolyn.

Ras-related protein 35 (Rab35) is a GTPase activated upstream of PI3K-Akt signaling ${ }^{110}$ that regulates neurite outgrowth in response to NGF by recruiting and complexing with MICAL-L1 and centaurinB2/ACAP2 to form a site on Arf6 + endosomes for EHD1 association ${ }^{111}$. It was later found that Rab35 serves as a master regulator of other downstream Rab proteins to bind with MICAL-L1 to modulate neurite outgrowth after NGF administration to PC12 cells ${ }^{112}$. Experiments with neuronal CAD cells show Rab35 is necessary for the formation of tunneling nanotubes ${ }^{113}$, protruding F-actin and tubulin cellular structures involved in vesicle trafficking and cell-to-cell communication, and macrophage inflammatory activation by LPS/IFN-y can impair their formation ${ }^{114}$. Rab35 plays multiple roles in vesicle trafficking that modulate the function and growth of neuronal protrusions and cromolyn significantly increases Rab35 secretion by TNF-a-induced HMC3 microglia. In conjunction with our neurite outgrowth experiments with PC12 cells, it is possible that the increased neurite length observed with cromolyn treatment beyond NGF treatment alone could be related to cromolyn promotion of Rab35 upstream of $\mathrm{PI} 3 \mathrm{~K} /$ Akt signaling.

Encouraging the phagocytic potential of microglia is crucial to maintaining anti-inflammatory clearance of damage associated molecular patterns, such as cellular debris of dead or dying cells, misfolded or aggregate proteins, and reduction of cell surface marker expression that signal inflammation from the local and peripheral environment ${ }^{115}$. In Alzheimer's disease, microglia colocalize with amyloid plaques primarily consisting of amyloid $\beta$-protein, a key activator of the inflammatory microglia phenotype, that is recognized by microglia via Fc receptors, Toll-like receptors, CD36, RAGE and scavenger receptors ${ }^{116}$. Amyloid plaques are known neurotoxic entities of AD. Amyloid beta is associated with increased reactive oxygen species (ROS) production ${ }^{117}$ and its aggregate states pair with synaptoxicity and neuronal loss of function with underlying inflammation ${ }^{118}$. The ability of CNS microglia to identify and remove aggregated $A \beta$ is a necessary component of an effective treatment strategy for $A D$. Cromolyn has been previously shown to upregulate the phagocytosis of $A \beta 42$ in BV2 microglial cells ${ }^{37}$. We found in the present study that the ability of cromolyn and F-cromolyn to promote iPSC-derived microglia to phagocytize $A \beta 42$ is upregulated relative to controls, further indicating that the cromolyn platform is able to influence microglia towards an anti-inflammatory phenotype. Figure 6 summarizes the proposed waypoints along the path to Alzheimer's disease that cromolyn and F-cromolyn are able to measurably affect.

\section{Conclusion}


Neuroinflammation is known to be a significant component of most neurological disorders - including ALS, MS, AD, Huntington's, and others. Chronic inflammation leads to increased neuronal damage that provides a positive feedback mechanism that prolongs inflammatory microglia activation to recruit leukocytes and other peripheral cells to the sites of damage instead of maintaining microglial clearance mechanisms. In the context of chronic inflammation, the body's wound healing process becomes damaging to brain function. Excessive ECM deposition and glial scarring can be obstructive to neurons and puts stress on their processes, inhibiting their ability to function normally. There is currently no approved treatment regimen available to tackle the multi-factorial nature of neurodegenerative disorders to slow or prevent disease. An efficacious therapeutic will be required to address disease-related triggers of plaque and tau tangles and of the neuroinflammatory response, fibrosis, as well as neurogenesis. Here we present evidence that cromolyn and F-cromolyn are able to dampen inflammation in HMC3 human microglia induced by TNF-a and promote secretion of anti-inflammatory IL-4. We also show that cromolyn and F-cromolyn reduce key components of the ECM, including fibronectin and tenascin-c, that have been related to fibrosis. Additionally, we show that cromolyn and F-cromolyn are able to augment NGF's capability to encourage neurite outgrowth of PC12 cells as well as promote phagocytosis of neurotoxic $A \beta 42$ by iPSC-derived microglia, and that cromolyn may achieve this by targeting PI3K signaling. Altogether, the cromolyn drug platform represents a robust treatment strategy for multiple aspects of neurodegenerative diseases that currently have no approved therapeutic.

\section{Declarations}

\section{Acknowledgements}

We gratefully acknowledge the continued support of AZTherapies, Inc.

\section{Competing Interests}

Y.W. and M.A.D. are employed by AZTherapies, Inc. S.C. and T.M.S. declare no competing interests. D.R.E. is an Associate Professor at Harvard Medical School and holds a part time employee position at Massachusetts General Hospital and serves as Chairmen at AZTherapies, Inc.

\section{Author Contributions}

Y.W. performed the lab work. S.C. and T.M.S contributed to preparation and testing of the drugs. Y.W. and M.A.D. constructed the figures and wrote the manuscript. D.R.E. lead the project.

\section{References}

1. Stephenson, J., Nutma, E., van der Valk, P. \& Amor, S. Inflammation in CNS neurodegenerative diseases.Immunology154,204-219(2018).

2. Tay, T. L., Savage, J. C., Hui, C. W., Bisht, K. \& Tremblay, M. Ã. Microglia across the lifespan: from origin to function in brain development, plasticity and cognition. The Journal of 
Physiology595,1929-1945(2017).

3. Chu, T. et al. Dynamic response of microglia/macrophage polarization following demyelination in mice.Journal of Neuroinflammation16,188(2019).

4. Colonna, M. \& Butovsky, O. Microglia Function in the Central Nervous System During Health and Neurodegeneration. Annu Rev Immunoß35,441-468(2017).

5. Michell-Robinson, M. A. et al. Roles of microglia in brain development, tissue maintenance and repair.Brain138,1138-1159(2015).

6. Crapser, J. D. et al. Microglia facilitate loss of perineuronal nets in the Alzheimer's disease brain.EBioMedicine58, (2020).

7. Nguyen, P. T. et al. Microglial Remodeling of the Extracellular Matrix Promotes Synapse Plasticity.Cel/182,388-403.e15(2020).

8. Wake, H., Moorhouse, A. J., Jinno, S., Kohsaka, S. \& Nabekura, J. Resting Microglia Directly Monitor the Functional State of Synapses In Vivo and Determine the Fate of Ischemic Terminals.J. Neurosci.29,3974(2009).

9. Szepesi, Z., Manouchehrian, O., Bachiller, S. \& Deierborg, T. Bidirectional Microglia-Neuron Communication in Health and Disease. Frontiers in Cellular Neuroscience12,323(2018).

10. Aldrich, A. \& Kielian, T. Central nervous system fibrosis is associated with fibrocyte-like infiltrates. Am $J$ Patho/179,2952-2962(2011).

11. Yang, T., Dai, Y., Chen, G. \& Cui, S. Dissecting the Dual Role of the Glial Scar and Scar-Forming Astrocytes in Spinal Cord Injury. Frontiers in Cellular Neuroscience14,78(2020).

12. Göritz, C. et al. A pericyte origin of spinal cord scar tissue.Science333,238-242(2011).

13. Dias, D. O. \& Göritz, C. Fibrotic scarring following lesions to the central nervous system.Matrix Bio/68-69,561-570(2018).

14. Jansson, D. et al. A role for human brain pericytes in neuroinflammation. Journal of Neuroinflammation11,104(2014).

15. Dong, J. \& Ma, Q. In Vivo Activation and Pro-Fibrotic Function of NF-kB in Fibroblastic Cells During Pulmonary Inflammation and Fibrosis Induced by Carbon Nanotubes. Frontiers in Pharmacology10,1140(2019).

16. Liddelow, S. A. et al. Neurotoxic reactive astrocytes are induced by activated microglia.Nature541,481-487(2017).

17. Clarke, L. E. et al. Normal aging induces A1-like astrocyte reactivity.Proc Natl Acad Sci USA115,E1896(2018).

18. Dorrier, C. E. et al. CNS fibroblasts form a fibrotic scar in response to immune cell infiltration. Nature Neuroscience24,234-244(2021).

19. Wang, Y. J., Monteagudo, A., Downey, M. A., Ashton-Rickardt, P. G. \& Elmaleh, D. R. Cromolyn inhibits the secretion of inflammatory cytokines by human microglia (HMC3).Scientific Reports11,8054(2021). 
20. Rappert, A. et al. CXCR3-Dependent Microglial Recruitment Is Essential for Dendrite Loss after Brain Lesion.J. Neurosci.24,8500(2004).

21. Qin, S. et al. The chemokine receptors CXCR3 and CCR5 mark subsets of T cells associated with certain inflammatory reactions. J Clin Invest101,746-754(1998).

22. Morris, A. W. J., Carare, R. O., Schreiber, S. \& Hawkes, C. A. The Cerebrovascular Basement Membrane: Role in the Clearance of $\beta$-amyloid and Cerebral Amyloid Angiopathy.Front Aging Neurosci6,251251(2014).

23. Reed, M. J., Damodarasamy, M. \& Banks, W. A. The extracellular matrix of the blood-brain barrier: structural and functional roles in health, aging, and Alzheimer's disease. Tissue Barriers7,1651157(2019).

24. Bikbaev, A., Frischknecht, R. \& Heine, M. Brain extracellular matrix retains connectivity in neuronal networks.Sci Rep5,14527(2015).

25. Végh, M. J. et al. Reducing hippocampal extracellular matrix reverses early memory deficits in a mouse model of Alzheimer's disease.Acta Neuropathologica Communications2,76(2014).

26. Lepelletier, F. X., Mann, D. M. A., Robinson, A. C., Pinteaux, E. \& Boutin, H. Early changes in extracellular matrix in Alzheimer's disease. Neuropathol Appl Neurobio/43,167-182(2017).

27. Milner, R. \& Campbell, I. L. The extracellular matrix and cytokines regulate microglial integrin expression and activation.J Immuno/170,3850-3858(2003).

28. Stoyanov, S. et al. Attenuation of the extracellular matrix restores microglial activity during the early stage of amyloidosis.Glia69,182-200(2021).

29. Haruwaka, K. et al. Dual microglia effects on blood brain barrier permeability induced by systemic inflammation. Nature Communications10,5816(2019).

30. Sofroniew, M. V. \& Vinters, H. V. Astrocytes: biology and pathology.Acta Neuropatho/119,7-35(2010).

31. Kigerl, K. A., de Rivero Vaccari, J. P., Dietrich, W. D., Popovich, P. G. \& Keane, R. W. Pattern recognition receptors and central nervous system repair.Exp Neuro/258,5-16(2014).

32. Valiente-Alandi, I. et al. Inhibiting Fibronectin Attenuates Fibrosis and Improves Cardiac Function in a Model of Heart Failure. Circulation138,1236-1252(2018).

33. Bhattacharyya, S. et al. Tenascin-C drives persistence of organ fibrosis. Nature Communications7,11703(2016).

34. Seo, M. C. et al. Discoidin domain receptor 1 mediates collagen-induced inflammatory activation of microglia in culture.J Neurosci Res86,1087-1095(2008).

35. Etich, J. et al. Gene Expression Profiling of the Extracellular Matrix Signature in Macrophages of Different Activation Status: Relevance for Skin Wound Healing.Int J Mol Sci20,5086(2019).

36. Hori, Y. et al. A Food and Drug Administration-approved asthma therapeutic agent impacts amyloid beta in the brain in a transgenic model of Alzheimer disease.J Biol Chem290,1966-1978(2015).

37. Zhang, C. et al. Cromolyn Reduces Levels of the Alzheimer's Disease-Associated Amyloid $\beta$-Protein by Promoting Microglial Phagocytosis.Scientific Reports8,1144(2018). 
38. Brazier, D., Perry, R., Keane, J., Barrett, K. \& Elmaleh, D. R. Pharmacokinetics of Cromolyn and Ibuprofen in Healthy Elderly Volunteers.Clin Drug Investig37,1025-1034(2017).

39. Shoup, T. M. et al. Evaluation of Fluorinated Cromolyn Derivatives as Potential Therapeutics for Alzheimer's Disease.J Alzheimers Dis80,775-786(2021).

40. Bruderer, R. et al. Optimization of Experimental Parameters in Data-Independent Mass Spectrometry Significantly Increases Depth and Reproducibility of Results.Mol Cell Proteomics16,22962309(2017).

41. Callister, S. J. et al. Normalization approaches for removing systematic biases associated with mass spectrometry and label-free proteomics.J Proteome Res5,277-286(2006).

42. Cox, T. R. \& Erler, J. T. Remodeling and homeostasis of the extracellular matrix: implications for fibrotic diseases and cancer.Dis Model Mech4,165-178(2011).

43. Gursoy, A., Keskin, O. \& Nussinov, R. Topological properties of protein interaction networks from a structural perspective.Biochem Soc Trans36,1398-1403(2008).

44. Ross, S. H. \& Cantrell, D. A. Signaling and Function of Interleukin-2 in T Lymphocytes.Annu Rev Immunoß36,411-433(2018).

45. Donnelly, R. P. et al. Tissue-specific regulation of IL-6 production by IL-4. Differential effects of IL-4 on nuclear factor-kappa B activity in monocytes and fibroblasts. J Immuno/151,5603-5612(1993).

46. Usenovic, M. et al. Internalized Tau Oligomers Cause Neurodegeneration by Inducing Accumulation of Pathogenic Tau in Human Neurons Derived from Induced Pluripotent Stem Cells.J. Neurosci.35,14234(2015).

47. Hughes, A. L., Gollapudi, L., Sladek, T. L. \& Neet, K. E. Mediation of Nerve Growth Factor-driven Cell Cycle Arrest in PC12 Cells by p53: SIMULTANEOUS DIFFERENTIATION AND PROLIFERATION SUBSEQUENT TO p53 FUNCTIONAL INACTIVATION *. Journal of Biological Chemistry275,3782937837(2000).

48. Li, S. et al. Histone deacetylase 1 promotes glioblastoma cell proliferation and invasion via activation of PI3K/AKT and MEK/ERK signaling pathways.Brain Research1692,154-162(2018).

49. Datta, M. et al. Histone Deacetylases 1 and 2 Regulate Microglia Function during Development, Homeostasis, and Neurodegeneration in a Context-Dependent Manner.Immunity48,514529.e6(2018).

50. Wylezinski, L. S. \& Hawiger, J. Interleukin 2 Activates Brain Microvascular Endothelial Cells Resulting in Destabilization of Adherens Junctions *. Journal of Biological Chemistry291,22913-22923(2016).

51. Zhao, X. et al. Neuronal Interleukin-4 as a Modulator of Microglial Pathways and Ischemic Brain Damage.J. Neurosci.35,11281(2015).

52. Zhang, K. et al. CXCL1 Contributes to $\beta$-Amyloid-Induced Transendothelial Migration of Monocytes in Alzheimer's Disease.PLOS ONE8,e72744(2013).

53. Johnson, E. A. et al. Increased expression of the chemokines CXCL1 and MIP-1a by resident brain cells precedes neutrophil infiltration in the brain following prolonged soman-induced status 
epilepticus in rats. Journal of Neuroinflammation8,41(2011).

54. Sawant, K. V. et al. Chemokine CXCL1 mediated neutrophil recruitment: Role of glycosaminoglycan interactions.Scientific Reports6,33123(2016).

55. Parajuli, B., Horiuchi, H., Mizuno, T., Takeuchi, H. \& Suzumura, A. CCL11 enhances excitotoxic neuronal death by producing reactive oxygen species in microglia.Glia63,2274-2284(2015).

56. Tarkowski, E. et al. Increased intrathecal levels of the angiogenic factors VEGF and TGF-beta in Alzheimer's disease and vascular dementia.Neurobiol Aging23,237-243(2002).

57. Chiappelli, M. et al. VEGF gene and phenotype relation with Alzheimer's disease and mild cognitive impairment. Rejuvenation Res9,485-493(2006).

58. Ding, X. et al. Microglia enhanced the angiogenesis, migration and proliferation of co-cultured RMECs.BMC Ophthalmo/18,249-249(2018).

59. Garbern, J. Y. et al. Patients lacking the major CNS myelin protein, proteolipid protein 1, develop length-dependent axonal degeneration in the absence of demyelination and inflammation.Brain125,551-561(2002).

60. Tatar, C. L. et al. Increased Plp1 Gene Expression Leads to Massive Microglial Cell Activation and Inflammation Throughout the Brain.ASN Neuro2,AN20100016(2010).

61. Thakkar, R. et al. PELP1: a key mediator of oestrogen signalling and actions in the brain. Journal of Neuroendocrinology30,e12484(2018).

62. Girard, B. J. et al. Cytoplasmic Localization of Proline, Glutamic Acid, Leucine-rich Protein 1 (PELP1) Induces Breast Epithelial Cell Migration through Up-regulation of Inhibitor of $\kappa B$ Kinase $\epsilon$ and Inflammatory Cross-talk with Macrophages.J Biol Chem292,339-350(2017).

63. Luo, Y. et al. PELP1 signaling contributes to medulloblastoma progression by regulating the NF-KB pathway.Molecular Carcinogenesis59,281-292(2020).

64. Langenfurth, A. et al. Membrane-type 1 metalloproteinase is upregulated in microglia/brain macrophages in neurodegenerative and neuroinflammatory diseases. Journal of Neuroscience Research92,275-286(2014).

65. Sareddy, G. R. \& Vadlamudi, R. K. PELP1: Structure, biological function and clinical significance.Gene585,128-134(2016).

66. Dou, F., Chang, X. \& Ma, D. Hsp90 Maintains the Stability and Function of the Tau Phosphorylating Kinase GSK3ß.Int J Mol Sci8,51-60(2007).

67. Sato, S., Fujita, N. \& Tsuruo, T. Modulation of Akt kinase activity by binding to Hsp90.Proc Natl Acad Sci U S A97,10832-10837(2000).

68. Giulino-Roth, L. et al. Inhibition of Hsp90 Suppresses PI3K/AKT/mTOR Signaling and Has Antitumor Activity in Burkitt Lymphoma.Mol Cancer Ther16,1779(2017).

69. Subbarao Sreedhar, A., Kalmár, Ã., Csermely, P. \& Shen, Y. F. Hsp90 isoforms: functions, expression and clinical importance. FEBS Letters562,11-15(2004). 
70. He, G. L. et al. Inhibition of HSP90ß by ganetespib blocks the microglial signalling of evoked proinflammatory responses to heat shock.Int J Biochem Cell Bio/106,35-45(2019).

71. Collins, C. B. et al. Targeted inhibition of heat shock protein 90 suppresses tumor necrosis factor-a and ameliorates murine intestinal inflammation. Inflamm Bowel Dis20,685-694(2014).

72. Hunter, M. C. et al. Hsp90 binds directly to fibronectin (FN) and inhibition reduces the extracellular fibronectin matrix in breast cancer cells.PLoS One9,e86842-e86842(2014).

73. Okada, M., Itoh, H., Hatakeyama, T., Tokumitsu, H. \& Kobayashi, R. Hsp90 is a direct target of the antiallergic drugs disodium cromoglycate and amlexanox. Biochem $3374,433-441$ (2003).

74. van Horssen, J. et al. Collagen XVIII: a Novel Heparan Sulfate Proteoglycan Associated with Vascular Amyloid Depositions and Senile Plaques in Alzheimer's Disease Brains. Brain Pathology12,456462(2002).

75. Al Ahmad, A. et al. Endostatin binds nerve growth factor and thereby inhibits neurite outgrowth and neuronal migration in-vitro.Brain Res1360,28-39(2010).

76. Hu, T. H. et al. Increased endostatin/collagen XVIII expression correlates with elevated VEGF level and poor prognosis in hepatocellular carcinoma.Modern Pathology18,663-672(2005).

77. Zhang, Y. \& Stefanovic, B. Akt mediated phosphorylation of LARP6; critical step in biosynthesis of type I collagen.Scientific Reports6,22597(2016).

78. Wegner, K. A. et al. Prostate epithelial-specific expression of activated PI3K drives stromal collagen production and accumulation.J Pathol250,231-242(2020).

79. Chen, W., Rock, J. B., Yearsley, M. M., Ferrell, L. D. \& Frankel, W. L. Different collagen types show distinct rates of increase from early to late stages of hepatitis C-related liver fibrosis. Hum Pathol45,160-165(2014).

80. Williams, L. M. et al. Identifying collagen $\mathrm{VI}$ as a target of fibrotic diseases regulated by CREBBP/EP300.Proc Natl Acad Sci USA117,20753(2020).

81. Li, X. H. et al. Gene Expression Profile of the Hippocampus of Rats Subjected to Chronic Immobilization Stress.PLOS ONE8,e57621(2013).

82. To, W. S. \& Midwood, K. S. Plasma and cellular fibronectin: distinct and independent functions during tissue repair.Fibrogenesis \& Tissue Repair4,21(2011).

83. Almutairi, M. M. A., Gong, C., Xu, Y. G., Chang, Y. \& Shi, H. Factors controlling permeability of the blood-brain barrier.Cell Mol Life Sci73,57-77(2016).

84. Sikkema, A. H. et al. Fibronectin aggregates promote features of a classically and alternatively activated phenotype in macrophages. Journal of Neuroinflammation15,218(2018).

85. Jones, H. et al. Inhibition of mast cell-secreted histamine decreases biliary proliferation and fibrosis in primary sclerosing cholangitis Mdr2-/- mice. Hepatology64,1202-1216(2016).

86. Hargrove, L. et al. Isolation and characterization of hepatic mast cells from cholestatic rats. Lab Invest96,1198-1210(2016). 
87. Wu, C., Keivenst, V. M., O'Toole, T. E., McDonald, J. A. \& Ginsberg, M. H. Integrin activation and cytoskeletal interaction are essential for the assembly of a fibronectin matrix.Cel/83,715-724(1995).

88. Stewart, R. L. \& O'Connor, K. L. Clinical significance of the integrin a6ß4 in human malignancies. Laboratory Investigation95,976-986(2015).

89. Sathyanesan, M. et al. A molecular characterization of the choroid plexus and stress-induced gene regulation. Translational Psychiatry2,e139-e139(2012).

90. Yang, Y. et al. Inflammation leads to distinct populations of extracellular vesicles from microglia. Journal of Neuroinflammation15,168(2018).

91. Richens, J. L. et al. Rationalising the role of Keratin 9 as a biomarker for Alzheimer's disease.Scientific Reports6,22962(2016).

92. Marzeda, A. M. \& Midwood, K. S. Internal Affairs: Tenascin-C as a Clinically Relevant, Endogenous Driver of Innate Immunity.J Histochem Cytochem.66,289-304(2018).

93. Mi, Z. et al. Tenascin-C Is Associated with Cored Amyloid- $\beta$ Plaques in Alzheimer Disease and Pathology Burdened Cognitively Normal Elderly.Journal of Neuropathology \& Experimental Neurology75,868-876(2016).

94. Haage, V. et al. Tenascin $\mathrm{C}$ regulates multiple microglial functions involving TLR4 signaling and HDAC1.Brain, Behavior, and Immunity81,470-483(2019).

95. Dong, H. et al. Suppression of Brain Mast Cells Degranulation Inhibits Microglial Activation and Central Nervous System Inflammation.Mol Neurobio/54,997-1007(2017).

96. Nong, Y. et al. Tenascin-C expression is associated with poor prognosis in hepatocellular carcinoma (HCC) patients and the inflammatory cytokine TNF-a-induced TNC expression promotes migration in HCC cells.Am J Cancer Res5,782-791(2015).

97. Kollins, K. M., Bell, R. L., Butts, M. \& Withers, G. S. Dendrites differ from axons in patterns of microtubule stability and polymerization during development. Neural Dev4,26-26(2009).

98. Park, J. H. \& Roll-Mecak, A. The tubulin code in neuronal polarity. Curr Opin Neurobio/51,95102(2018).

99. Erck, C. et al. A vital role of tubulin-tyrosine-ligase for neuronal organization. Proc Natl Acad Sci USA102,7853(2005).

100. Song, W., Cho, Y., Watt, D. \& Cavalli, V. Tubulin-tyrosine Ligase (TTL)-mediated Increase in Tyrosinated a-Tubulin in Injured Axons Is Required for Retrograde Injury Signaling and Axon Regeneration.J Biol Chem290,14765-14775(2015).

101. Lee, J. et al. H. A post-transcriptional program coordinated by CSDE1 prevents intrinsic neural differentiation of human embryonic stem cells.Nature Communications8,1456(2017).

102. Guo, H. et al. Disruptive variants of CSDE1 associate with autism and interfere with neuronal development and synaptic transmission.Sci Adv5,eaax2166(2019).

103. Youn, J. Y. et al. High-Density Proximity Mapping Reveals the Subcellular Organization of mRNAAssociated Granules and Bodies.Mol Cel/69,517-532.e11(2018). 
104. Hu, S., Claud, E. C., Musch, M. W. \& Chang, E. B. Stress granule formation mediates the inhibition of colonic Hsp70 translation by interferon-y and tumor necrosis factor-a.American Journal of Physiology-Gastrointestinal and Liver Physiology298,G481-G492(2010).

105. Wolozin, B. \& Ivanov, P. Stress granules and neurodegeneration. Nature Reviews Neuroscience20,649-666(2019).

106. Kaltezioti, V. et al. Prox1 regulates the notch1-mediated inhibition of neurogenesis.PLoS Bio/8,e1000565(2010).

107. Ia Poulsen, L. et al. C. Inhibition of Endothelial NOTCH1 Signaling Attenuates Inflammation by Reducing Cytokine-Mediated Histone Acetylation at Inflammatory Enhancers.Arteriosclerosis, Thrombosis, and Vascular Biology38,854-869(2018).

108. Kato, K. et al. Prox1 Inhibits Proliferation and Is Required for Differentiation of the Oligodendrocyte Cell Lineage in the Mouse.PLOS ONE10,e0145334(2016).

109. Kaltezioti, V. et al. Prox 1 inhibits neurite outgrowth during central nervous system development. Cellular and Molecular Life Sciences78,3443-3465(2021).

110. Wheeler, D. B., Zoncu, R., Root, D. E., Sabatini, D. M. \& Sawyers, C. L. Identification of an oncogenic RAB protein.Science350,211(2015).

111. Kobayashi, H. \& Fukuda, M. Rab35 establishes the EHD1-association site by coordinating two distinct effectors during neurite outgrowth.J Cell Sci126,2424-2435(2013).

112. Kobayashi, H., Etoh, K., Ohbayashi, N. \& Fukuda, M. Rab35 promotes the recruitment of Rab8, Rab13 and Rab36 to recycling endosomes through MICAL-L1 during neurite outgrowth.Biology Open3,803814(2014).

113. Bhat, S. et al. Rab35 and its effectors promote formation of tunneling nanotubes in neuronal cells.Scientific Reports10,16803(2020).

114. Goodman, S., Naphade, S., Khan, M., Sharma, J. \& Cherqui, S. Macrophage polarization impacts tunneling nanotube formation and intercellular organelle trafficking.Scientific Reports9,14529(2019).

115. Westman, J., Grinstein, S. \& Marques, P. E. Phagocytosis of Necrotic Debris at Sites of Injury and Inflammation. Frontiers in Immunology10,3030(2020).

116. Doens, D. \& Fernández, P. L. Microglia receptors and their implications in the response to amyloid $\beta$ for Alzheimer's disease pathogenesis. Journal of Neuroinflammation11,48(2014).

117. Cheignon, C. et al. Oxidative stress and the amyloid beta peptide in Alzheimer's disease.Redox Bio/14,450-464(2018).

118. Joshi, P. et al. Microglia convert aggregated amyloid- $\beta$ into neurotoxic forms through the shedding of microvesicles. Cell Death \& Differentiation21,582-593(2014).

\section{Figures}



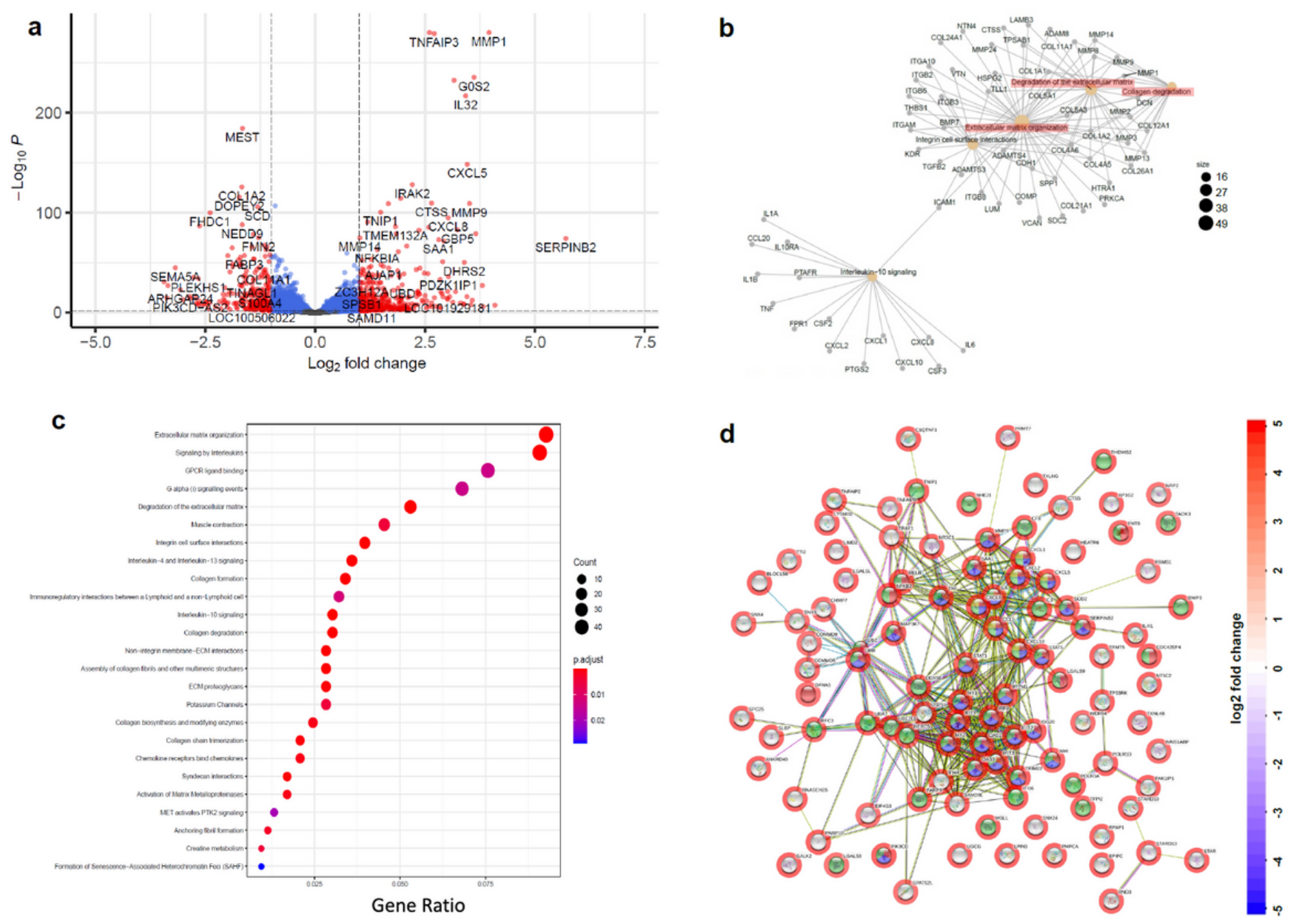

\section{Figure 1}

TNF-a potently induces differential expression of a wide array of fibrosis and inflammation-associated genes and proteins. (a) Volcano plot includes 26,485 genes quantified, with red dots showing gene candidates. 1061 genes are differentially expressed upon addition of $0.3 \mu \mathrm{g} / \mathrm{mL}$ TNF-a to HMC3 microglia. Cutoff is log2 fold change ( $>2$ folds) and $p$-value $<0.05$. (b) Function-to-Gene association Network (FGNet) plot presents genes significantly altered by TNF-a as they relate to the indicated cellular function. (c) Pathway enrichment dotplot collects pathway associations among significantly altered genes after TNF-a administration. The dot size represents the number of genes related to the indicated pathway whose expressions were significantly changed and its color indicates its statistical significance. (d) String-db functional analysis of Protein-Protein Interaction Network (PPINet). Each spherical node is a protein significantly changed after TNF-a administration to HMC3 cells, with the color aura of the sphere indicating log2 fold change from HMC3 cells alone. Each sphere is annotated by color: red for response to cytokines, blue for cytokine-mediated signaling pathway, green for response to stress, and yellow for chemokine-mediated signaling pathway. Each edge represents a PPI, either experimentally determined or known from curated databases; or the edge represents predicted protein interactions based on genomic or proteomic neighborhood, fusions, or co-occurrence. 

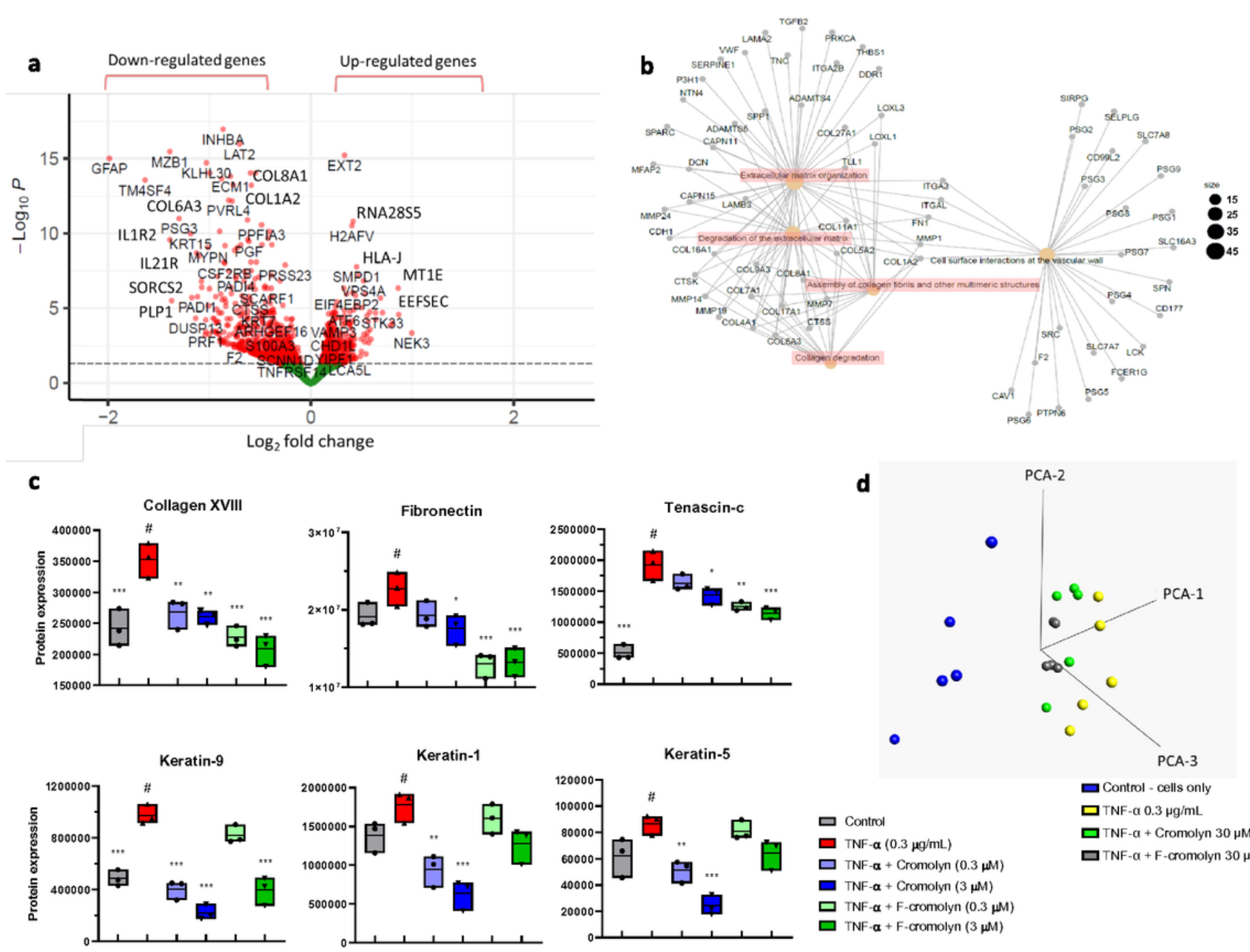

PCA-2

\section{Figure 2}

Cromolyn reduces expression of fibrosis and inflammation-associated genes and proteins induced by TNF-a in HMC3 human microglia. (a) Volcano plot showing $30 \mu \mathrm{M}$ cromolyn addition to $0.3 \mu \mathrm{g} / \mathrm{mL}$ TNF-a induced HMC3 microglia differentially changed expression of 1441 genes with cutoff $p$-value $<0.05$. (b) FGNet analysis shows that $30 \mu \mathrm{M}$ cromolyn addition to TNF-a treated HMC3 microglia affects the indicated cellular function. (c) Cromolyn and F-cromolyn significantly reduce fibrosis-associated protein secretion by TNF-a-induced HMC3 microglia, including collagen XVIII, fibronectin, and tenascin-c, and keratin-9, -1 , and -5 . Quantitative analysis of secreted proteins relative to the \# group of TNF-a alone. Oneway ANOVA was used for statistics: ${ }^{\star \star \star} p<0.001,{ }^{\star \star} p<0.01,{ }^{\star} p<0.05$ versus the \# group. (d) 3D principal component analysis (PCA) shows cromolyn and F-cromolyn significantly alter TNF-a-induced collective gene expression in HMC3 microglia as compared to the cells alone. The plotted points are condensed representations of gene expression $0.3 \mu \mathrm{g} / \mathrm{mL}$ TNF-a treatment cluster (yellow) being most spatially distant from the control cluster (blue). $30 \mu \mathrm{M}$ Cromolyn (green) and $30 \mu \mathrm{M}$ F-cromolyn (grey) groups are clustered together. 


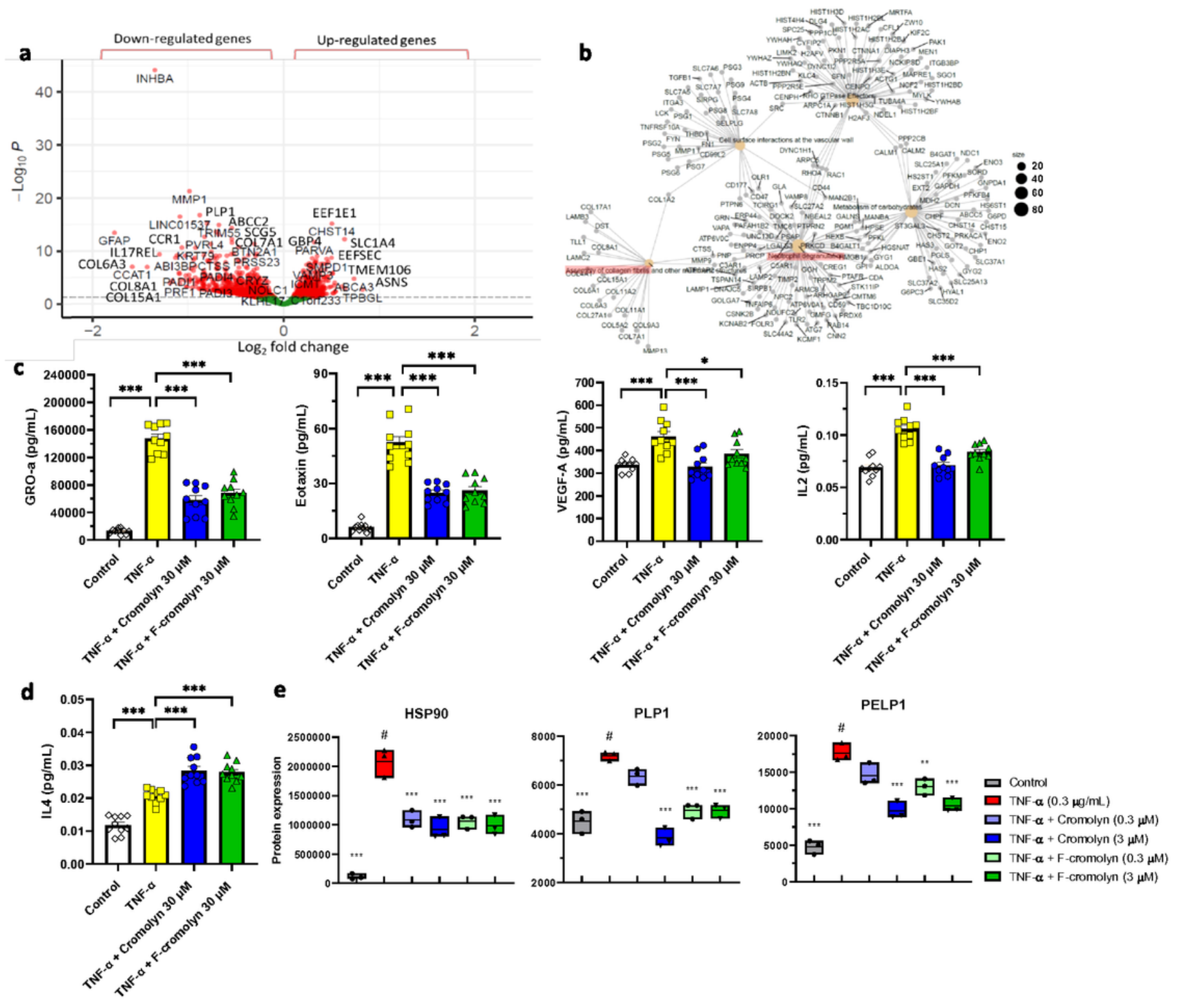

Figure 3

F-cromolyn reduces expression of fibrosis- and inflammation-associated genes and proteins induced by TNF-a in HMC3 human microglia. (a) Volcano plot of 2108 genes differentially altered by $30 \mu \mathrm{M} \mathrm{F-}$ cromolyn after $0.3 \mu \mathrm{g} / \mathrm{mL}$ TNF-a addition. (b) FGNet analysis of cellular pathways significantly affected by F-cromolyn. Dot size is indicative of the approximate number of genes grouped with a particular cellular pathway. (c) Cytokine secretion of HMC3 microglia controls (white diamonds), after addition of $0.3 \mu \mathrm{g} / \mathrm{mL}$ TNF-a (yellow squares), $0.3 \mu \mathrm{g} / \mathrm{mL}$ TNF- $\mathrm{a}+30 \mu \mathrm{M}$ cromolyn (blue circles), and $0.3 \mu \mathrm{g} / \mathrm{mL}$ TNF-a $+30 \mu \mathrm{M}$ F-cromolyn (green triangles) for GRO-a, Eotaxin, VEGF-A, IL-2, and (d) IL-4. ${ }^{\star \star \star} \mathrm{p}<0.001$, $\star \star p<0.01,{ }^{\star} p<0.05$. (e) Cromolyn and F-cromolyn significantly reduce pro-inflammatory protein secretion by TNF-a-induced HMC3 microglia, including HSP90, PLP1, and PELP1. Quantitative analysis of secreted proteins relative to \# group of TNF-a alone. One-way ANOVA was used for statistics: ${ }^{\star \star \star} p<0.001$, ${ }^{\star \star} \mathrm{p}<$ $0.01,{ }^{*} p<0.05$ versus the \# group. 


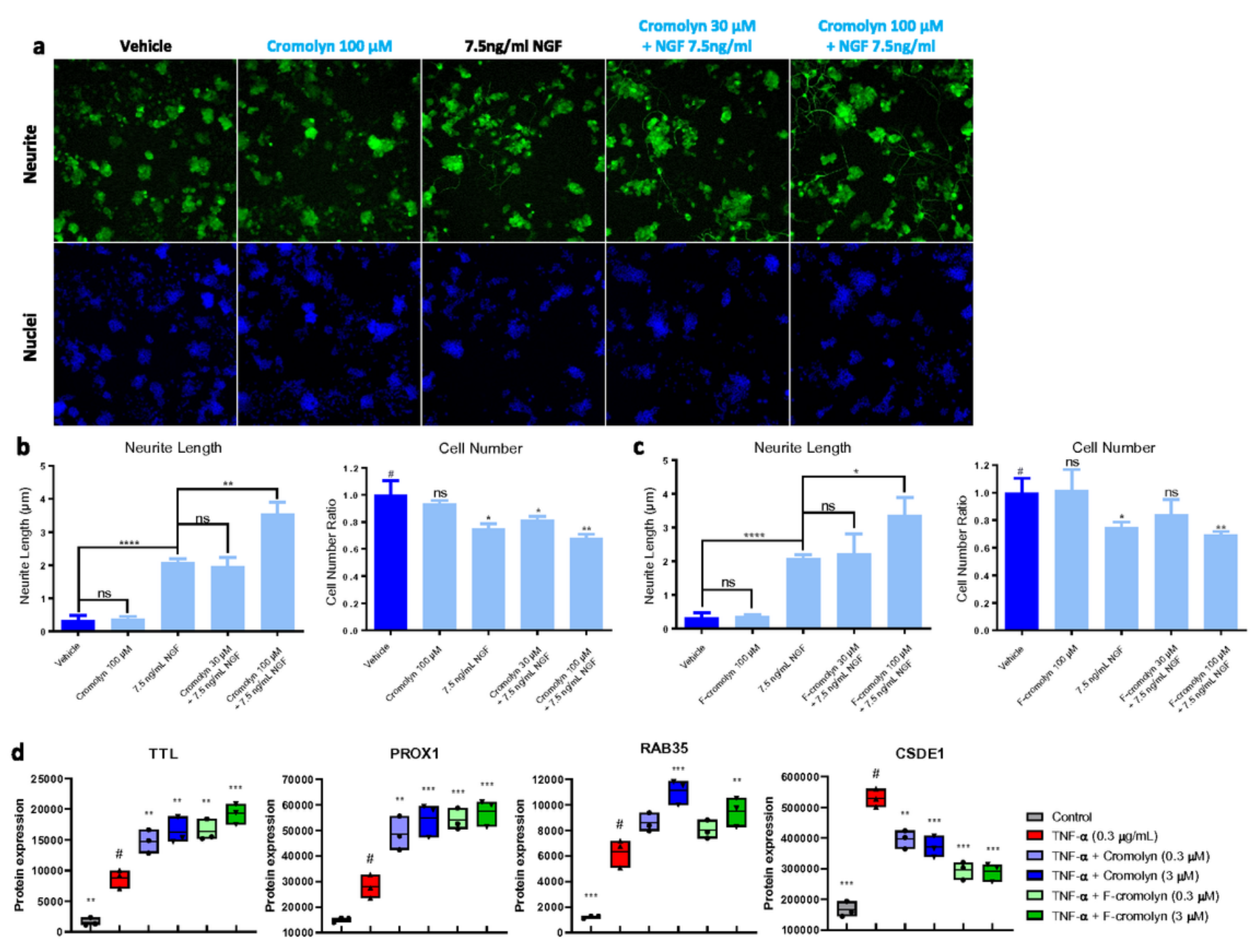

\section{Figure 4}

Cromolyn and F-cromolyn promote neurite outgrowth and neurogenesis in PC12 cells and differentially alter neurogenesis-related proteins in HMC3 microglia. (a) Top row incorporates PC12 cell staining with Alexa Fluor 488 for $\beta 3$-tubulin (green) for imaging of neurite outgrowth over vehicle, $100 \mu \mathrm{M}$ cromolyn, $7.5 \mathrm{ng} / \mathrm{mL}$ nerve growth factor (NGF), $30 \mu \mathrm{M}$ cromolyn $+7.5 \mathrm{ng} / \mathrm{mL}$ NGF, and $100 \mu \mathrm{M}$ cromolyn +7.5 $\mathrm{ng} / \mathrm{mL}$ NGF conditions. Bottom row incorporates Hoechst staining for nuclei of PC12 cells for comparison over the same treatment conditions. (b) Neurite length bar plots and cell number measurements normalized to vehicle for PC12 cells over indicated treatment condition, including (b) cromolyn and (c) F-cromolyn. ${ }^{* \star \star} \mathrm{p}<0.0001,{ }^{* \star} \mathrm{p}<0.01,{ }^{\star} \mathrm{p}<0.05$, ns $=$ no significance. (d) Cromolyn and F-cromolyn significantly regulate the expression of neurite outgrowth and neurogenesis-associated proteins by TNF-a-induced HMC3 microglia, including TTL, PROX1, RAB35, and CSDE1. Quantitative analysis of secreted proteins relative to \# group of TNF-a alone. One-way ANOVA was used for statistics: $\star \star \star * p<0.001,{ }^{* \star} \mathrm{p}<0.01,{ }^{\star} \mathrm{p}<0.05$ versus the \# group. 
a

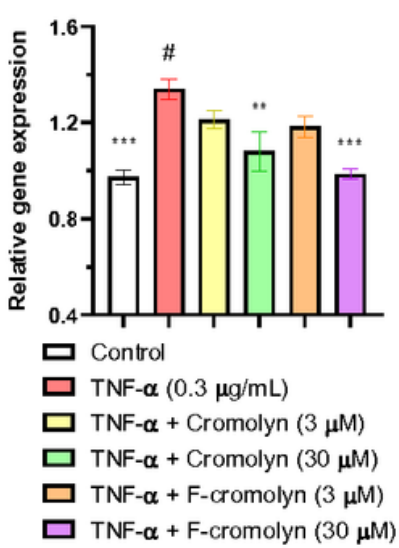

b

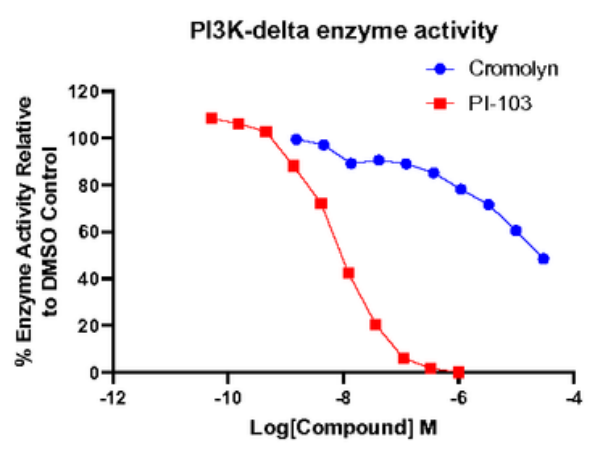

C

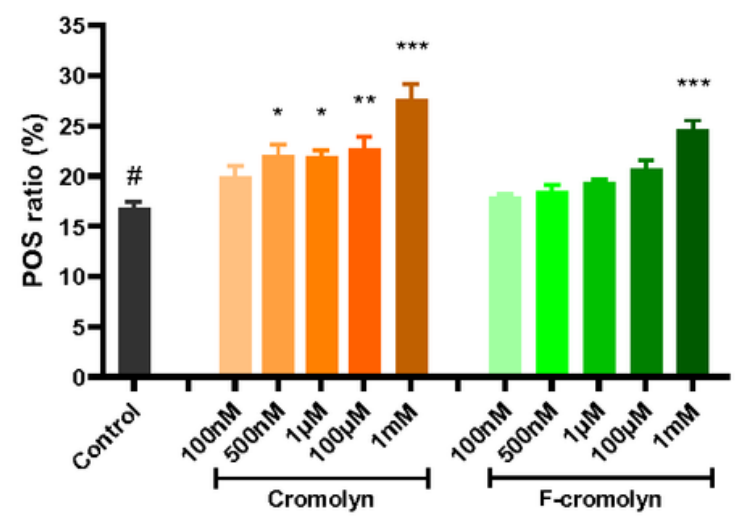

d

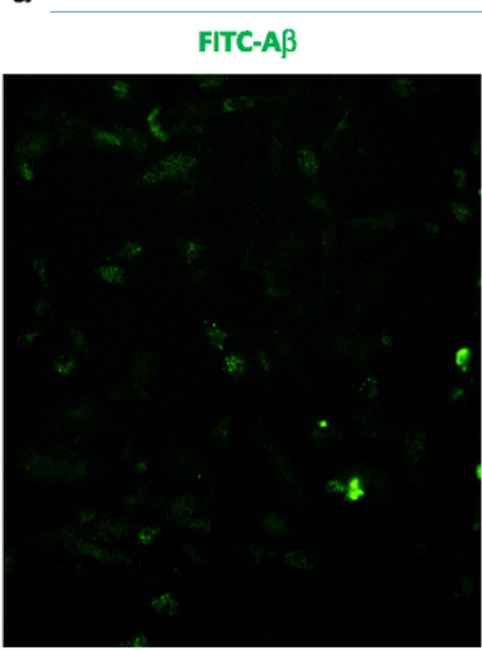

Control

FITC-A Lysotracker Hoechst

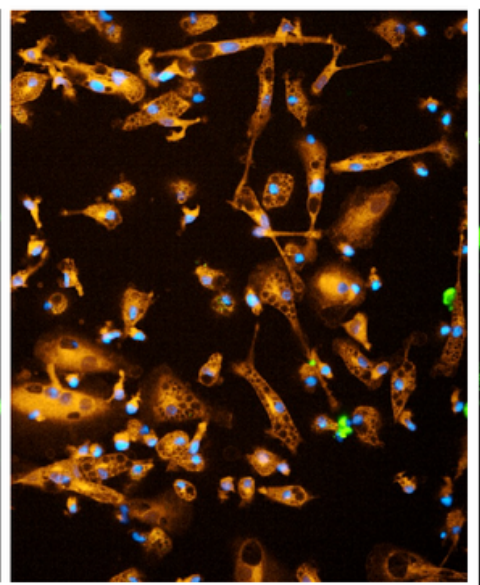

e

Cromolyn, $1 \mathrm{mM}$

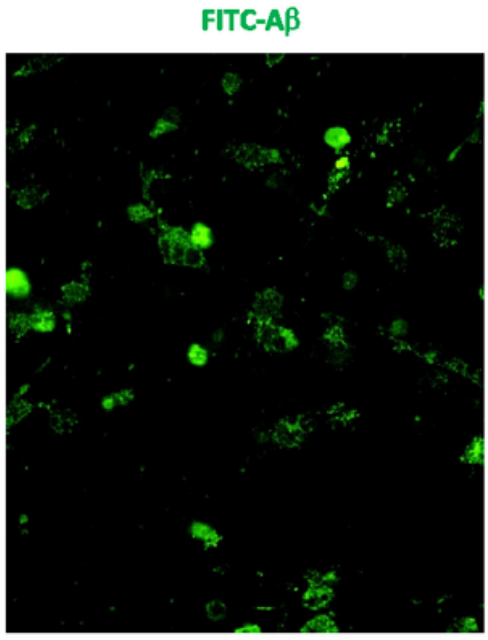

FITC-A $\beta$ Lysotracker Hoechst

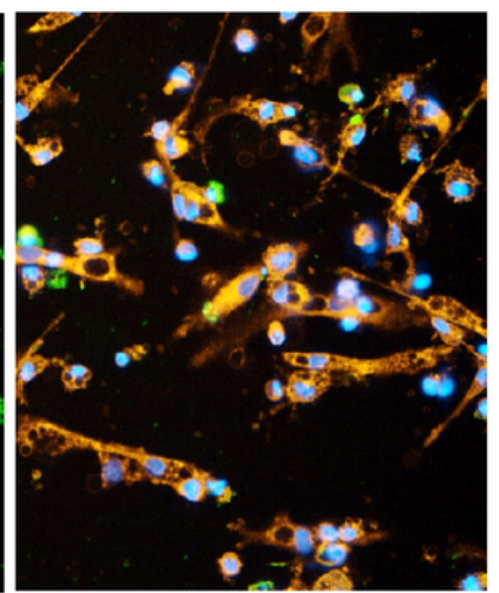

Figure 5

Cromolyn and F-cromolyn promote iPSC-derived human microglial phagocytosis of amyloid $\beta$-protein 42 (Aß42) via PI3K signaling. (a) RNA-seq analysis show that cromolyn and F-cromolyn decreased the gene expression levels of PIK3CD induced by TNF-a in HMC3 human microglia. Fold change is normalized to untreated control. GAPDH was used as internal control to normalize mRNA level of the gene of measurement in each sample. Quantitative analysis of PIK3CD gene expression relative to \# group of TNF-a alone. One-way ANOVA was used for statistics: ${ }^{*} P<0.05$, ${ }^{\star *} P<0.01$, $* \star * P<0.001$ versus to \# group. (b) Cromolyn inhibited the activity of PI3K- $\delta$ lipid kinase in a concentration-dependent manner. Compound $\mathrm{PI}-103$ is used as positive control inhibitor of PI3K- $\delta$. (c) Comparison of microglia internalization of FITC-A $\beta$ at indicated concentrations for both cromolyn and F-cromolyn. POS ratio $=$ (cell number with phagocytosed $A \beta 42$ )/(total cell number). ${ }^{*} P<0.05$, ${ }^{\star *} P<0.01$, ${ }^{\star * *} P<0.001$. (d) (left) Control image with fluorescein labeled A 342 (FITC-A $\beta$ ) with human microglia and (right) an overlay image with FITC-A $\beta$ (green), lysotracker for cell lysosome (orange), and Hoechst nuclei stain (blue). (e) $1 \mathrm{mM}$ cromolyn treatment to microglia. (left) image of FITC-A $\beta$ only and (right) overlay image with FITC-A $\beta$ (green), lysotracker for cell lysosome (orange), and Hoechst nuclei stain (blue). 


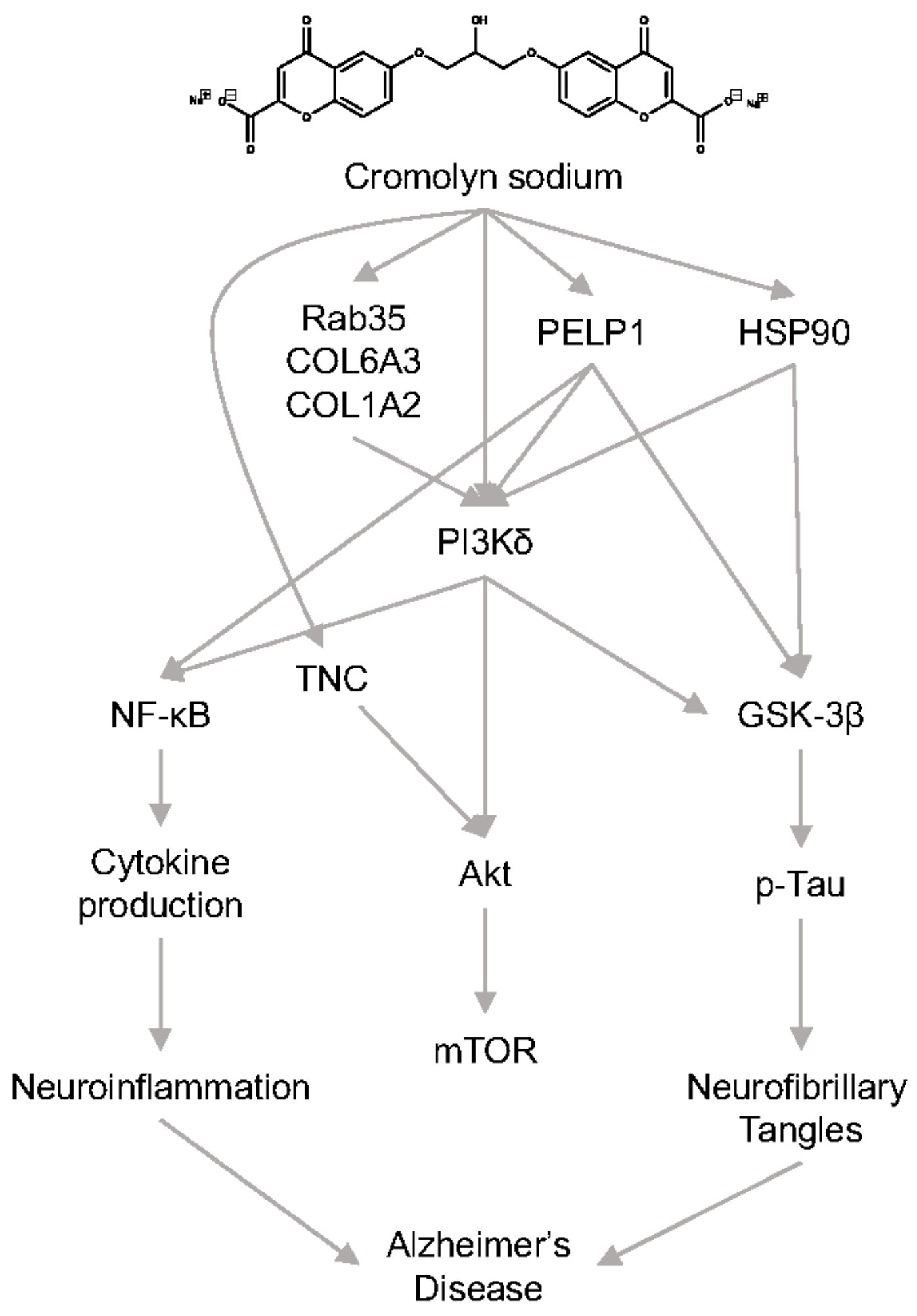

Figure 6

Hypothesis for the upstream modification by cromolyn and F-cromolyn to significantly affect many factors along the way to Alzheimer's disease.

\section{Supplementary Files}


This is a list of supplementary files associated with this preprint. Click to download.

- Supplementarylnfo.docx 